\title{
QUANTIZATION OF KÄHLER MANIFOLDS. II
}

\author{
MICHEL CAHEN, SIMONE GUTT AND JOHN RAWNSLEY
}

\begin{abstract}
We use Berezin's dequantization procedure to define a formal *product on a dense subalgebra of the algebra of smooth functions on a compact homogeneous Kähler manifold $\boldsymbol{M}$. We prove that this formal $*$-product is convergent when $M$ is a hermitian symmetric space.
\end{abstract}

\section{INTRODUCTION}

In part I of this paper [7] we showed how to quantize certain compact Kähler manifolds $(M, \omega, J)$. This means the following: Let $(L, \nabla, h)$ be a quantization bundle over $M$ (i.e., a holomorphic line bundle $L$ with connection $\nabla$ admitting an invariant hermitian structure $h$, such that the curvature is $\operatorname{curv}(\nabla)=-2 i \pi \omega)$. Let $\mathscr{H}$ be the Hilbert space of holomorphic sections of $L$. To any linear operator $A$ on $\mathscr{H}$ is associated a symbol $\hat{A}$ which is a real analytic function on $M$. Denote by $\hat{E}(L)$ the space of these symbols. For any positive integer $k,\left(L^{k}=\bigotimes^{k} L, \nabla^{(k)}, h^{(k)}\right)$ is a quantization bundle for $(M, k \omega, J)$. If $\mathscr{C}^{k}$ is the Hilbert space of holomorphic sections of $L^{k}$, we denote by $\hat{E}\left(L^{k}\right)$ the space of symbols of linear operators on $\mathscr{Z}^{k}$. If, for every $k$, a certain characteristic function $\varepsilon^{(k)}$ (which depends on $L$ and $k$ and which is real analytic on $M)$ is constant, the space $\hat{E}\left(L^{l}\right)$ is contained in the space $\hat{E}\left(L^{k}\right)$ for any $k \geq l$. Furthermore $\bigcup_{l=1}^{\infty} \hat{E}\left(L^{l}\right)$ (denoted by $\mathscr{C}_{L}$ ) is a dense subspace of the space of continuous functions on $M$. Any function $f$ in $\mathscr{C}_{L}$ belongs to a particular $\hat{E}\left(L^{l}\right)$ and is thus the symbol of an operator $A_{f}^{(k)}$ acting on $\mathscr{H}^{k}$ for $k \geq l$. One has thus constructed, for a given $f$, a family of quantum operators parametrized by an integer $k$.

From the point of view of deformation theory [1], where quantization is realised at the level of the algebra of functions, one can say that one has constructed a family of associative products on $\hat{E}\left(L^{l}\right)$, with values in $\mathscr{C}_{L}$, parametrized by an integer $k$; indeed

$$
f *_{k} g=A_{f}^{(\widehat{k)}} A_{g}^{(k)}, \quad f, g \in \hat{E}\left(L^{l}\right), k \geq l .
$$

The aim of part II is twofold. Firstly, we prove that, for any compact generalized flag manifold, there exists on $\mathscr{C}_{L}$ a formal differential $*$-product with

Received by the editors May 22, 1990.

1980 Mathematics Subject Classification (1985 Revision). Primary 58F06.

Key words and phrases. Quantization, Kähler manifolds.

The second author is a Research Associate of the National Fund for Scientific Research (Belgium). 
parameter $\nu$ which coincides with the asymptotic expansion of the previously defined $*_{k}$ associative products when $\nu=i /(4 \pi k)$. Secondly, we prove that, when $M$ is a hermitian symmetric space, the $*_{k}$-product of two functions in $\mathscr{C}_{L}$ is a rational function of $k$ having no pole at infinity. Hence the formal *-product is in fact convergent.

The paper is organised as follows: In $\S 1$ we study the geometrical properties of two 2-point functions on $M$, one of which is related to Calabi's diastatic function [3]. In $\S 2$ we prove the existence of an asymptotic expansion in $k^{-1}$ of the $*_{k}$-product of two elements of $\hat{E}\left(L^{l}\right)$. In $\S 3$ we turn to flag manifolds and prove that the asymptotic expansion constructed in $\S 2$ defines a formal *-product. The proof depends crucially both on the homogeneity and on the density property proved in part I [7]. Finally $\S 4$ is devoted to the proof of the rational dependence in $\nu$ of the associative product of two functions in $\mathscr{C}_{L}$ when $M$ is hermitian symmetric.

The relationship between quantization by deformation and a calculus of symbols has been studied by many authors and in particular by F. Berezin [2] and by $C$. Moreno [6]. We hope that results presented here help to elucidate some of the underlying geometry and the difficulties which have to be overcome to understand completely the compact Kähler case.

Acknowledgment. We are pleased to thank our friend Joe Wolf who gave us the benefit of his expertise in the geometry of hermitian symmetric spaces. We also thank the British Council and the Communauté française de Belgique for their support during the preparation of this work.

\section{The Relationship between Calabi's diastatic Functions AND LINE BUNDLES}

The formula for the product of symbols (to be described in $\S 2$ ) introduces a 2-point function $\psi$ defined in terms of coherent states. Some of the properties of this function are described here. Another, somewhat related 2-point function $\widetilde{\psi}$, defined locally in terms of nonzero holomorphic sections, turns out to be expressible in terms of Calabi's diastatic function $D$. In this section we study these three functions $D, \psi, \widetilde{\psi}$ and in particular we give a formula for the Hessian of such a function at a critical point.

As in [7], $(M, \omega, J)$ will be a compact Kähler manifold and $(L, \nabla, h)$ a quantization bundle over $M$. Consider a real-analytic, closed, real 2-form $\Omega$ on $M$ of type $(1,1)$. On a contractible open set $U \subset M$ there exists a real 1 -form $\beta$ such that $\Omega=d \beta$. One may write $\beta=\alpha+\bar{\alpha}$ for some $(1,0)$-form $\alpha$ on $U$. Since $\Omega$ is type $(1,1)$

$$
\Omega=(\partial+\bar{\partial})(\alpha+\bar{\alpha})=\partial \alpha+(\bar{\partial} \alpha+\partial \bar{\alpha})+\bar{\partial} \bar{\alpha}
$$

implies $\partial \alpha=0$. Thus, by the Dolbeault lemma, there exists a function $g$ defined on a possibly smaller open set $V \subset U$, such that

$$
\Omega=\bar{\partial} \partial g+\partial \bar{\partial} \bar{g}=\partial \bar{\partial}(\bar{g}-g)=(i / 4 \pi) \partial \bar{\partial} f
$$

where $f$ is the real-valued function $f=4 \pi i(g-\bar{g})$. The function $f$ is not unique: if $\partial \bar{\partial} f_{1}=0, \partial f_{1}$ is a holomorphic $(1,0)$-form, thus there exists locally a holomorphic function $h$ such that $\partial f_{1}=\partial h=d h$. The reality of $f_{1}$ implies that $\bar{\partial} f_{1}=d \bar{h}$ and thus $d f_{1}=d(h+\bar{h})$; i.e., $f_{1}$ is the real part of a holomorphic function. 
A function $f$ satisfying (1.1) is called a potential for $\Omega$. Since $\Omega$ is real analytic, $f$ is also real analytic and may thus be complex-analytically continued to an open neighbourhood $W$ of the diagonal in $V \times \bar{V}$. Denote this extension by $f(x, \bar{y})$. It is holomorphic in $x$, antiholomorphic in $y$ and, with this notation, $f(x)=f(x, \bar{x})$.

Consider the function $D_{(\Omega)}: W \rightarrow \mathbb{C}$,

$$
D_{(\Omega)}(x, y)=f(x, \bar{x})+f(y, \bar{y})-f(x, \bar{y})-f(y, \bar{x}) .
$$

Since $f$ is real valued on $V$, one has $\overline{f(x, \bar{y})}=f(y, \bar{x})$ and thus $D_{(\Omega)}$ is real valued. One checks that $D_{(\Omega)}$ does not depend on the choice of the local potential $f$ and is thus a globally defined function on a neighbourhood of the diagonal in $M \times \bar{M}$, depending only on $\Omega$. It is called the Calabi function of $\Omega$.

Observe that, for any $y \in M$, the set $U_{y}=\left\{x \in M \mid D_{(\Omega)}(x, y)\right.$ is defined $\}$ is a neighbourhood of $y$. Denote by $D_{(\Omega) y}$ the function on $U_{y}$ defined by $D_{(\Omega) y}(x)=D_{(\Omega)}(x, y)$, then $\partial \bar{\partial} D_{(\Omega) y}(x)=(\partial \bar{\partial} f)(x, \bar{x})$ and thus $D_{(\Omega) y}$ is a potential for $\Omega$ on $U_{y}$.

Definition 1. Calabi's diastatic function $D$ is the Calabi function of the Kähler form $\omega$.

Example 1. Let $M=\mathbb{C}^{n}$ and let $\omega=(i / 2) \sum_{j=1}^{n} d z_{j} \wedge d \bar{z}_{j}$ be the canonical Kähler form, then a potential $f$ is given by

$$
f=2 \pi \sum_{j=1}^{n}\left|z_{j}\right|^{2}
$$

and the Calabi diastatic function $D$ is

$$
\begin{aligned}
D\left(z, z^{\prime}\right) & =2 \pi \sum_{j=1}^{n}\left[\left|z_{j}\right|^{2}+\left|z_{j}^{\prime}\right|^{2}-z_{j} \bar{z}_{j}^{\prime}-z_{j}^{\prime} \bar{z}_{j}\right] \\
& =2 \pi \sum_{j=1}^{n}\left|z_{j}-z_{j}^{\prime}\right|^{2} .
\end{aligned}
$$

It is thus, up to a factor, the square of the distance between the points $z$ and $z^{\prime}$.

Example 2. Let $M=\mathbb{C} P^{n}$ and let $\pi: \mathbb{C}^{n+1} \backslash\{0\} \rightarrow \mathbb{C} P^{n}$ be the canonical projection. Denote by $\langle$,$\rangle the standard metric on \mathbb{C}^{n+1}$. The Fubini-Study metric $g$ on $\mathbb{C} P^{n}$ is such that

$$
\left(\pi^{*} g\right)_{z}(X, Y)=\frac{1}{\pi\|z\|^{4}}\left[\langle X, Y\rangle\|z\|^{2}-\left\langle X, A_{z}\right\rangle\left\langle Y, A_{z}\right\rangle-\left\langle X, B_{z}\right\rangle\left\langle Y, B_{z}\right\rangle\right]
$$

where $z \in \mathbb{C}^{n+1}$ and

$$
A_{z}=\sum_{\alpha \leq n}\left(z_{\alpha} \partial_{z_{\alpha}}+\bar{z}_{\alpha} \partial_{\bar{z}_{\alpha}}\right), \quad B_{z}=\sum_{\alpha \leq n}\left(-i z_{\alpha} \partial_{z_{\alpha}}+i \bar{z}_{\alpha} \partial_{\bar{z}_{\alpha}}\right) .
$$

Considering the chart $U=\left\{p \mid p=\pi(z)\right.$ with $\left.z^{n+1} \neq 0\right\}$ and the coordinate map $\phi: U \rightarrow \mathbb{C}^{n}, \phi(p)=u$ where $p=\pi(z)$ and $u_{j}=z^{j} / z^{n+1}, j \leq n$, one 
sees that the Kähler form $\omega$ on $U$ has the form

$$
\omega=\frac{i}{2 \pi\left(1+\sum_{\lambda}\left|u_{\lambda}\right|^{2}\right)^{2}}\left[\left(1+\sum_{\lambda}\left|u_{\lambda}\right|^{2}\right) \sum_{\alpha} d u_{\alpha} \wedge d \bar{u}_{\alpha} \sum_{\alpha, \beta} \bar{u}_{\alpha} d u_{\alpha} \wedge u_{\beta} d \bar{u}_{\beta}\right] .
$$

Hence a Kähler potential $f$ is given by

$$
f=2 \log \left(1+\sum_{\lambda}\left|u_{\lambda}\right|^{2}\right)
$$

In these coordinates the diastatic function has the expression

$$
\begin{aligned}
D\left(u, u^{\prime}\right)=2[\log (1+ & \left.\sum_{\lambda}\left|u_{\lambda}\right|^{2}\right)+\log \left(1+\sum_{\lambda}\left|u_{\lambda}^{\prime}\right|^{2}\right) \\
& \left.-\log \left(1+\sum_{\lambda} u_{\lambda} \bar{u}_{\lambda}^{\prime}\right)-\log \left(1+\sum_{\lambda} u_{\lambda}^{\prime} \bar{u}_{\lambda}\right)\right] .
\end{aligned}
$$

This can be rewritten in terms of the coordinates in $\mathbb{C}^{n+1}$ as

$$
D\left(\pi(z), \pi\left(z^{\prime}\right)\right)=2 \log \frac{\|z\|^{2}\left\|z^{\prime}\right\|^{2}}{\left|\left\langle z, z^{\prime}\right\rangle\right|^{2}} .
$$

In particular $D>0$ unless $\pi(z)=\pi\left(z^{\prime}\right)$, where $D=0$.

Observe that $\mathbb{C}^{n+1} \backslash\{0\}$ may be identified with a principal $\mathbb{C}^{*}$-bundle over $\mathbb{C} P^{n}$. Observe also that the argument of the logarithm in formula (1.4) may be rewritten for any hermitian line bundle by using local sections. These observations will lead us to the definition of our second 2-point function.

Let $\pi: L \rightarrow M$ be a holomorphic line bundle with real-analytic hermitian structure $h$. Let $s: U \rightarrow L$ be a zero-free holomorphic section of $L$ over the open set $U \subset M$. Then $|s|^{2}(x)=h_{x}(s(x), s(x))$ is a real-analytic function on $U$ which can be analytically continued to a neighbourhood of the diagonal in $U \times \bar{U}$, to give a function $|s|^{2}(x, \bar{y})$ holomorphic in $x$ and antiholomorphic in $y$. This function has nonzero values for $y$ sufficiently close to $x$. Consider then the expression (analogous to the one in (1.4))

$$
\widetilde{\psi}(x, y)=\frac{|s|^{2}(x, \bar{x})|s|^{2}(y, \bar{y})}{\left.\left.|| s\right|^{2}(x, \bar{y})\right|^{2}}
$$

wherever this is defined, which will be the case in a neighbourhood of the diagonal. Remark that if $t: U \rightarrow L$ is another holomorphic section on $U$ without 0 , there exists a holomorphic function $f: U \rightarrow \mathbb{C}$ such that $t=f \cdot s$. Then

$$
\frac{|t|^{2}(x, \bar{x})|t|^{2}(y, \bar{y})}{\left.\left.|| t\right|^{2}(x, \bar{y})\right|^{2}}=\frac{|f(x)|^{2}|s|^{2}(x, \bar{x})|f(y)|^{2}|s|^{2}(y, \bar{y})}{\left.\left.|f(x) \bar{f}(y)|^{2}|| s\right|^{2}(x, \bar{y})\right|^{2}}=\widetilde{\psi}(x, y) .
$$

This justifies the following definition.

Definition 2. If $L \rightarrow M$ is a holomorphic line bundle with real-analytic hermitian structure $h$, the 2-point function $\widetilde{\psi}$ defined locally by formula (1.5), in a neighbourhoood of the diagonal in $M \times \bar{M}$, will be called the characteristic function of the bundle $L$, and denoted $\widetilde{\psi}_{L}$.

Before considering some of the properties of $\widetilde{\psi}$, let us exhibit the relationship between $\widetilde{\psi}$ and Calabi's function. 
Proposition 1. Let $\pi: L \rightarrow M$ be a holomorphic line bundle with real analytic hermitian structure $h$. Let $\nabla$ be the unique connection, [5], on L leaving $h$ invariant and such that $\nabla_{X} s=0$ for any $X$ of type $(0,1)$ and for any local holomorphic section $s$. Let $\omega=(i / 2 \pi) \operatorname{curv}(\nabla)$. Then

$$
\widetilde{\psi}=e^{-\frac{1}{2} D}
$$

where $D$ is the Calabi function of $\omega$.

Remark. If $(L, \nabla, h)$ is a quantization bundle for $M$, the Kähler form satisfies the assumption of Proposition 1 and thus $\widetilde{\psi}=e^{-\frac{1}{2} D}$ where $D$ is Calabi's diastatic function.

Proof. Let $s: U \rightarrow L$ be a holomorphic section of $L$. Then

$$
\nabla_{X} s=\alpha_{s}(X) s
$$

where $\alpha_{s}$ is a 1 -form of type $(1,0)$. Also

$$
d|s|^{2}=\left(\alpha_{s}+\bar{\alpha}_{s}\right)|s|^{2}
$$

and thus

$$
\alpha_{s}=\partial \log |s|^{2} .
$$

The curvature 2-form $\sigma$ is defined by

$$
\sigma(X, Y) s=\left(\nabla_{X} \nabla_{Y}-\nabla_{Y} \nabla_{X}-\nabla_{[X, Y]}\right) s
$$

and for $s$ holomorphic we get

$$
\sigma(X, Y) s=\left(d \alpha_{s}\right)(X, Y) s .
$$

That is

$$
\sigma=\bar{\partial} \partial \log |s|^{2}=-\partial \bar{\partial} \log |s|^{2} .
$$

Thus for $\omega=(i / 2 \pi) \sigma,-2 \log |s|^{2}$ is a potential and we can compute the Calabi function $D$ of $\omega$ as

$$
D(x, y)=2 \log \left[\frac{|s|^{2}(x, \bar{y})|s|^{2}(y, \bar{x})}{|s|^{2}(x, \bar{x})|s|^{2}(y, \bar{y})}\right]=-2 \log \widetilde{\psi}(x, y),
$$

hence the conclusion.

The 2-point function has the following two properties: (a) Let $\pi: L \rightarrow M$, $\pi^{\prime}: L^{\prime} \rightarrow M$ be two holomorphic hermitian line bundles and let $\widetilde{\psi}_{L}$ and $\widetilde{\psi}_{L^{\prime}}$ be the corresponding characteristic functions. If $s: U \rightarrow L$ and $s^{\prime}: U \rightarrow L^{\prime}$ are local sections then $s \otimes s^{\prime}$ is a local section of $L \otimes L^{\prime}$. Hence

$$
\widetilde{\psi}_{L \otimes L^{\prime}}=\widetilde{\psi}_{L} \cdot \widetilde{\psi}_{L^{\prime}}
$$

In particular if one considers the $k$ th power $L^{k}$ of the bundle $L$, one has

$$
\widetilde{\psi}_{L^{k}}=\left(\widetilde{\psi}_{L}\right)^{k}
$$

(b) Let $f: M \rightarrow N$ be a holomorphic map and let $\pi: L \rightarrow N$ be a holomorphic hermitian line bundle. Then $f^{*} L \rightarrow M$ has natural holomorphic and hermitian structures such that if $s: U \rightarrow L$ is a local holomorphic section of $L$ over the open set $U \subset N$, then $s \circ f$, the corresponding section of $f^{*} L$ over $f^{-1} U$, is holomorphic and $|s \circ f|^{2}=|s|^{2} \circ f$. Formula (1.5) shows that

$$
\widetilde{\psi}_{f * L}=\widetilde{\psi}_{L} \circ f .
$$

These properties justify the name characteristic function. 
The 2-point function $\widetilde{\psi}_{L}$ may be expressed in terms of coherent states and of the function $\varepsilon$ which has been introduced in part I and denoted by $\theta$ there. Denote by $\mathscr{H}_{L}$ the Hilbert space of holomorphic sections of $L$ with the scalar product

$$
\left\langle s, s^{\prime}\right\rangle=\int_{M} h_{x}\left(s(x), s^{\prime}(x)\right) \frac{\omega^{n}(x)}{n !}
$$

where $h$ is the hermitian structure of $L, \omega$ the Kähler form of $M$ and $\operatorname{dim} M=2 n$.

Evaluation of a holomorphic section $s$ at a point $x$ of $M$ is a continuous linear map $\mathscr{H}_{L} \rightarrow L_{x}=\pi^{-1}(x)$. Choose a base-point $q \in L_{x}$. Then there exists a unique element $e_{q} \in \mathscr{H}_{L}$, called a coherent state, such that

$$
s(x)=\left\langle s, e_{q}\right\rangle q, \quad x=\pi(q) .
$$

Since $e_{c q}=\bar{c}^{-1} e_{q}$ for $c$ a nonzero complex number, the function

$$
\varepsilon(x)=\left\|e_{q}\right\|^{2}|q|^{2}, \quad x=\pi(q)
$$

is well defined.

If $s_{0}: U \rightarrow L$ is a zero-free holomorphic section over $U$, and $s$ an arbitrary holomorphic section of $L$ then

$$
s(x)=\left\langle s, e_{s_{0}(x)}\right\rangle s_{0}(x), \quad x \in U .
$$

Thus $\left\langle s, e_{s_{0}(x)}\right\rangle$ is a holomorphic function of $x$ and hence $e_{s_{0}(x)}$ depends antiholomorphically on $x$. In particular

$$
\varepsilon(x)=\left\langle e_{s_{0}(x)}, e_{s_{0}(x)}\right\rangle\left|s_{0}(x)\right|^{2}
$$

is real analytic and admits an analytic extension to a neighbourhood of the diagonal in $U \times \bar{U}$, holomorphic in the first variable and antiholomorphic in the second

$$
\varepsilon(x, \bar{y})=\left\langle e_{s_{0}(y)}, e_{s_{0}(x)}\right\rangle\left|s_{0}\right|^{2}(x, \bar{y}) .
$$

In particular

$$
\begin{aligned}
\widetilde{\psi}(x, y) & =\frac{\varepsilon(x, \bar{x}) \varepsilon(y, \bar{y})}{\left\|e_{s_{0}(x)}\right\|^{2}\left\|e_{s_{0}(y)}\right\|^{2}} \frac{\left|\left\langle e_{s_{0}(y)}, e_{s_{0}(x)}\right)\right|^{2}}{|\varepsilon(x, \bar{y})|^{2}} \\
& =\frac{\varepsilon(x) \varepsilon(y)}{|\varepsilon(x, \bar{y})|^{2}} \frac{\left|\left\langle e_{q^{\prime}}, e_{q}\right\rangle\right|^{2}}{\left\|e_{q^{\prime}}\right\|^{2}\left\|e_{q}\right\|^{2}}, \quad q \in L_{x} \backslash\{0\}, q^{\prime} \in L_{y} \backslash\{0\} .
\end{aligned}
$$

We have seen in [7], the importance of the condition $\varepsilon$ being constant. This motivates the following definition.

Definition 3. A hermitian holomorphic line bundle $\pi: L \rightarrow M$ will be said to be regular if the function $\varepsilon$ is constant.

Definition 4. Let $\left\{e_{q} \mid q \in L_{0}\right\}$ be the set of coherent states of the hermitian line bundle $\pi: L \rightarrow M$. The 2-point function $\psi$ is defined by

$$
\psi(x, y)=\frac{\left|\left\langle e_{q^{\prime}}, e_{q}\right\rangle\right|^{2}}{\left\|e_{q^{\prime}}\right\|^{2}\left\|e_{q}\right\|^{2}}, \quad \pi(q)=x, \quad \pi\left(q^{\prime}\right)=y .
$$


$\psi$ is globally defined on $M \times M$ and takes values in $[0,1]$. In particular all points of the diagonal are critical points where $\psi$ takes the value 1 . Rewriting the definition in terms of a local holomorphic section $s_{0}$ we have

$$
\psi(x, y)=\frac{\left|\left\langle e_{s_{0}(x)}, e_{s_{0}(y)}\right\rangle\right|^{2}}{\left\|e_{s_{0}(x)}\right\|^{2}\left\|e_{s_{0}(y)}\right\|^{2}}
$$

which shows that $\psi$ is real analytic.

Reformulating the above, we have the next proposition.

Proposition 2. If $L$ is a regular, hermitian, holomorphic line bundle, the characteristic function $\widetilde{\psi}$ equals the 2-point function $\psi$ and is thus globally defined on $M \times \bar{M}$.

A precise result concerning the zeroes of the diastatic function (or equivalently the set of points in $M \times \bar{M}$ where $\widetilde{\psi}=1$ ) can be obtained for regular bundles which are sufficiently positive.

In this situation let $\mathscr{H}$ be the Hilbert space of holomorphic sections of $L$ and let $\phi: M \rightarrow P\left(\mathscr{H}^{*}\right), x=\pi(q) \mapsto \mathbb{C} l_{q}$ where $q \in L_{0}$ and $l_{q}(s) q=$ $\left\langle s, e_{q}\right\rangle q=s(x)$ for any $s \in \mathscr{H}$. When the Chern class $c_{1}(L)$ is sufficiently positive, this map $\phi$ is an embedding. Using $\S 3$ of [7], one sees that the pullback of $H^{*}$, the dual of the tautological bundle over $P\left(\mathscr{H}^{*}\right)$, is isomorphic to the given bundle $L$. Hence $\widetilde{\psi}_{L}=\widetilde{\psi}_{H^{*}} \circ \phi$. From Proposition 1 and from the expression of $D$ for complex projective space given in Example 2, one sees that $\psi(x, y)=\widetilde{\psi}_{L}(x, y)=1$ if and only if $x=y$. This proves the following proposition.

Proposition 3. If the bundle $L$ is regular and sufficiently positive, the diastatic function $D(x, y)$ vanishes if and only if $x=y$.

The function $\psi$ admits the points of the diagonal as critical points. In fact at these points $\psi$ has its maximum value, namely 1 , and if the bundle is sufficiently positive, $\psi(x, y)=1$ only if $x=y$. Choosing a point $x$ of $M$, we plan to compute the Hessian of the function $\psi(x,$.$) at y=x$.

If, as above, $H$ denotes the tautological bundle over $P\left(\mathscr{H}^{*}\right)$, then the hermitian form on $H$ is given by $z \mapsto|z|^{2}, z \in \mathscr{H}^{*} \backslash\{0\}$. Thus on the dual bundle $H^{*}$ it is given by $z \mapsto 1 /|z|^{2}$. Hence we get from (1.4) and Proposition 1

$$
\widetilde{\psi}_{H^{*}}(\phi(x), \phi(y))=\frac{\left|\left\langle l_{q}, l_{q^{\prime}}\right\rangle\right|^{2}}{\left\|l_{q}\right\|^{2}\left\|l_{q^{\prime}}\right\|^{2}}=\frac{\left|\left\langle e_{q^{\prime}}, e_{q}\right\rangle\right|^{2}}{\left\|e_{q}\right\|^{2}\left\|e_{q^{\prime}}\right\|^{2}}, \quad \phi(x)=\mathbb{C} l_{q},
$$

if $q$ (resp. $q^{\prime}$ ) belongs to $L_{0_{x}}$ (resp. $L_{0_{y}}$ ). This means that $\phi^{*} \widetilde{\psi}_{H^{*}}=\psi_{L}$. Observe that at a critical point $(x, x)$ of $\psi_{L}$, one has

$$
\left(\operatorname{Hess} \psi_{L}\right)_{(x, x)}(X, Y)=\operatorname{Hess} \widetilde{\psi}_{H^{*}}\left(\phi_{*} X, \phi_{*} Y\right)_{(\phi(x), \phi(y))} \text {. }
$$

The right-hand side can be computed readily:

$$
\left(\operatorname{Hess}_{2} \widetilde{\psi}_{H^{*}}\right)=-\pi g
$$

where the suffix 2 means that one fixes the first variable (and thus $\tilde{\psi}_{H^{*}}$ becomes a function on $P\left(\mathscr{H}^{*}\right)$ ) and where $g$ is the Fubini-Study metric given in Example 2. For $L$ sufficiently positive we then have the following proposition. 
Proposition 4. If $\pi: L \rightarrow M$ is a sufficiently positive regular bundle, the Hessian of the characteristic function $\psi_{L}$ (considered as a function of its second argument only) is given bv

$$
\operatorname{Hess}_{2} \psi_{L}=-\pi \phi^{*} g .
$$

In particular it is a nonsingular symmetric bilinear form.

Remark. Using the proposition of $\S 3$ of [7] one sees that the Hessian is proportional to the metric of $M$.

\section{THE COMPOSITION OF OPERATORS AND AN ASYMPTOTIC FORMULA}

As in $\S 1$, we denote by $(L, \nabla, h)$ a quantization bundle of the compact Kähler manifold $(M, \omega, J)$ and by $\mathscr{H}$ the Hilbert space of holomorphic sections of $L$. By compactness of $M$, it is finite dimensional. Let $A: \mathscr{H} \rightarrow \mathscr{H}$ be a linear operator and let

$$
\hat{A}(x)=\frac{\left\langle A e_{q}, e_{q}\right\rangle}{\left\langle e_{q}, e_{q}\right\rangle}, \quad q \in L_{0_{x}}, x \in M,
$$

be its symbol.

The composition of operators on $\mathscr{H}$ gives rise to a product for the corresponding symbols, which is associative and which we shall denote by $*$ following Berezin. For the basic facts about $*$ quantization see [1]. We have

$$
\begin{aligned}
(\hat{A} * \hat{B})(x) & =\widehat{A B}(x)=\frac{\left\langle A B e_{q}, e_{q}\right\rangle}{\left\|e_{q}\right\|^{2}}=\frac{\left\langle B e_{q}, A^{*} e_{q}\right\rangle}{\left\|e_{q}\right\|^{2}} \\
& =\int_{M} h_{y}\left(\left(B e_{q}\right)(y),\left(A^{*} e_{q}\right)(y)\right) \frac{1}{\left\|e_{q}\right\|^{2}} \frac{\omega^{n}(y)}{n !} \\
& =\int_{M}\left\langle B e_{q}, e_{q^{\prime}}\right\rangle\left\langle A e_{q^{\prime}}, e_{q}\right\rangle \frac{\left|q^{\prime}\right|^{2}}{\left\|e_{q}\right\|^{2}} \frac{\omega^{n}(y)}{n !} \\
& =\int_{M} \hat{A}(x, y) \hat{B}(y, x) \frac{\left|\left\langle e_{q^{\prime}}, e_{q}\right\rangle\right|^{2}}{\left\|e_{q^{\prime}}\right\|^{2}\left\|e_{q}\right\|^{2}} \varepsilon(y) \frac{\omega^{n}(y)}{n !} \\
& =\int_{M} \hat{A}(x, y) \hat{B}(y, x) \psi(x, y) \varepsilon(y) \frac{\omega^{n}(y)}{n !} .
\end{aligned}
$$

Let $k$ be a positive integer. The bundle $\left(L^{k}=\bigotimes^{k} L, \nabla^{k}, h^{k}\right)$ is a quantization bundle for $(M, k \omega, J)$ and we denote by $\mathscr{H}^{k}$ the corresponding space of holomorphic sections and by $\hat{E}\left(L^{k}\right)$ the space of symbols of linear operators on $\mathscr{H}^{k}$. We have proven in [7] the following facts (i) When $\varepsilon^{(k)}$ is constant for all $k$ (i.e., when all bundles $L^{k}$ are regular) one has the nesting property $\hat{E}\left(L^{k}\right) \subset \hat{E}\left(L^{k+1}\right)$.

(ii) With the same assumption $\bigcup_{k} \hat{E}\left(L^{k}\right)$ is dense in $\mathscr{C}^{0}(M)$. From $\S 1$, we recall that formula (1.8) and Proposition 2 prove that

$$
\psi^{(k)}(x, y)=(\psi(x, y))^{k} .
$$

From formula (2.1), the nesting property and formula (2.2) one sees that if $A, B$ belong to $\hat{E}\left(L^{l}\right)$ and if $k \geq l$ one may define

$$
\left(\hat{A} *_{k} \hat{B}\right)(x)=\int_{M} \hat{A}(x, y) \hat{B}(y, x) \psi^{k}(x, y) \varepsilon^{(k)} \frac{k^{n} \omega^{n}}{n !} .
$$


Remark 1. Let $G$ be a Lie group of isometries of the Kähler manifold $(M, \omega, J)$ which lifts to a group of automorphisms of the quantization bundle $(L, \nabla, h)$. This automorphism group acts naturally on the bundles $\left(L^{k}, \nabla^{(k)}, h^{k}\right)$. We have proven in [7] that if $g \in G$ and if $e_{q}^{(l)}$ is a coherent state of $L^{l}$, then $g . e_{q}^{(l)}=e_{g q}^{(l)}$. From this one deduces that for any $\hat{A}, \hat{B}$ in $\hat{E}\left(L^{l}\right)$ and any $k \geq l:$

$$
g^{*}\left(\hat{A} *_{k} \hat{B}\right)(x)=\left(\hat{A} *_{k} \hat{B}\right)(g x)=\left(\left(\widehat{g^{-1}} A g *_{k} \widehat{g^{-1} B} g\right)(x)=\left(g^{*} \hat{A} * g^{*} \hat{B}\right)(x)\right.
$$

which means that the product $*_{k}$ is $G$-invariant in the geometrical sense [1].

It is also a consequence of [7], that if $\mathfrak{g}$ is the Lie algebra of $G$ and $X \in$ $\mathfrak{g}$, the symbol of the quantum operator associated to the function $\lambda_{X}$, where $i\left(X^{*}\right) \omega=d \lambda_{X}$ and $X^{*}$ is the fundamental vector field on $M$ corresponding to $X$, is precisely that function $\lambda_{X}$. Hence the product $*_{k}$ is covariant, [1].

We plan to analyze the $k$-dependence of the formula (2.3). The first step in this direction is the rationality of $\varepsilon^{(k)}$.

Proposition 1. Let $(M, \omega, J)$ be a flag manifold, let $(L, \nabla, h)$ be a quantization bundle for $(M, \omega, J)$ and let $L^{k}=\bigotimes^{k} L$ and let $\varepsilon^{(k)}$ be the $\varepsilon$-function of the bundle $L^{k}$. Then $\varepsilon^{(k)}$ is a rational function of $k$, with no pole at infinity.

Proof. Recall that by the theorem of $\S 3$ of [7]

$$
\varepsilon^{(k)}=\frac{1}{(\operatorname{vol} M) k^{n}} \operatorname{dim} \mathscr{H}^{(k)}
$$

where $n=\frac{1}{2} \operatorname{dim} M$ and $\mathscr{H}^{(k)}$ is the space of holomorphic sections of $L^{k}$. Now $\mathscr{H}^{(k)}$ carries an irreducible representation of $G(M=G / K$ where $G$ is a compact connected, simply-connected Lie group and $K$ is the centralizer of a torus $T_{1}$ of $G$ ). Denote by $\lambda$ the highest weight of this representation. If $\mu$ is the highest weight of the irreducible representation of $G$ on $\mathscr{H}$, one has $\lambda=k \mu$.

Let $\mathfrak{g}$ be the Lie algebra of $G$ and $t$ be the algebra of a maximal torus $T \supseteq T_{1}$. Let $\Delta$ be the set of roots of $\mathfrak{g}^{\mathbb{C}}$ relative to $\mathfrak{t}^{\mathbb{C}}$. There exists an "admissible" Weyl chamber $C$ of $i t^{*}$ such that if $\Delta_{+}$is the set of positive roots relative to $C$ and if $\Phi$ is the set of positive simple roots, then $\Phi=\Phi_{1} \cup \Phi_{2}$ where $\Phi_{1}=\left\{\alpha \in \Phi \mid \alpha_{\mid \mathfrak{t}_{1}}=0\right\}$. Furthermore the algebra $\mathfrak{k}$ of the isotropy group $K$ is such that

$$
\mathfrak{k}^{\mathbb{C}}=\mathfrak{t}^{\mathbb{C}} \oplus \sum_{\alpha \in \Delta_{1}^{+}}\left(\mathfrak{g}^{\alpha}+\mathfrak{g}^{-\alpha}\right)
$$

where the elements of $\Delta_{1}^{+}$are sums of elements of $\Phi_{1}$. Denote by $\Delta_{2}^{+}=\Delta^{+} \backslash \Delta_{1}^{+}$. One has then

$$
\begin{aligned}
\operatorname{dim}_{\mathbb{R}} G / K & =\operatorname{dim} G-\operatorname{dim} \mathfrak{t}-2 \#\left\{\alpha \in \Delta_{1}^{+}\right\}, \\
n & =\frac{1}{2}(\operatorname{dim} G-\operatorname{dim} \mathfrak{t})-\#\left\{\alpha \in \Delta_{1}^{+}\right\} .
\end{aligned}
$$

Recall that Weyl's dimension formula tells us that the dimension $d_{\lambda}$ of $\mathscr{H}$ is given by

$$
d_{\lambda}=\frac{\prod_{\alpha \in \Delta^{+}}\langle\lambda+\delta, \alpha\rangle}{\prod_{\alpha \in \Delta^{+}}\langle\delta, \alpha\rangle}
$$

where $\delta$ denotes half the sum of the positive roots and $\langle$,$\rangle is the scalar$ product on $i t^{*}$ induced by the Killing form of $\mathfrak{g}$. 
Thus $d_{\lambda}$ is a polynomial in $k$ with degree $q$ equal to the number of positive roots which are not orthogonal to $\lambda$. Clearly $q=\#\left\{\alpha \in \Delta_{2}^{+}\right\}$. Indeed the stabilizer of the highest weight is equal to $K$ as the geometrical quantization conditions are satisfied. Hence the conclusion since $q=n$.

Remark 2. This proposition generalizes to the compact regular case. Indeed, in this situation $L \rightarrow M$ is a holomorphic, hermitian line bundle with connection $\nabla$ and the curvature of $\nabla$ is $2 \pi i \omega$. Thus $\omega$ is an integral form representing the first Chern class $c_{1}(L)$. As $\omega$ is a positive $(1,1)$-form, $c_{1}(L)>0$ and thus $L$ is an ample bundle. So passing to a sufficiently high power $k$ of $L$, Kodaira's map $\phi$ is an embedding of $M$ in projective space. In particular by Chow's theorem, $M$ is a projective algebraic variety.

Now by the Riemann-Roch-Hirzebruch formula the Euler-Poincare characteristic $\chi$ of the $\bar{\partial}$-complex is given by an integral of a polynomial in $c_{1}(L)$ of degree equal to the dimension of $M$. Kodaira's vanishing theorem replaces the Borel-Weil-Bott theorem to tell us that, if $c_{1}(L)$ is sufficiently positive (i.e. for $k$ sufficiently large), all cohomology spaces vanish in positive dimension. Hence $\chi$ reduces to $\operatorname{dim} \mathscr{H}\left(L^{k}\right)$ and is thus a polynomial of degree $\operatorname{dim} M$ in $k$.

Remark 3. The integral in (2.3) is an absolutely convergent integral which makes sense for any real number $k \geq l$. Indeed it may be rewritten as

$$
\begin{gathered}
\int_{M} \frac{\left\langle A e_{q^{\prime}}^{(l)}, e_{q}^{(l)}\right\rangle}{\left\langle e_{q^{\prime}}^{(l)}, e_{q}^{(l)}\right\rangle} \frac{\left\langle B e_{q}^{(l)}, e_{q^{\prime}}^{(l)}\right\rangle}{\left\langle e_{q}^{(l)}, e_{q^{\prime}}^{(l)}\right\rangle} \frac{\left\|e_{q^{\prime}}^{(l)}\right\|^{2}\left\|e_{q}^{(l)}\right\|^{2}}{\left\|e_{q^{\prime}}^{(l)}\right\|^{2}\left\|e_{q}^{(l)}\right\|^{2}} \frac{\left|q^{l}\right|^{2}\left|q^{\prime l}\right|^{2}}{\left|q^{l}\right|^{2}\left|q^{\prime l}\right|^{2}} \psi^{k}(x, y) \varepsilon^{(k)} \frac{k^{n} \omega^{n}}{n !} \\
\quad=\int_{M}\left\langle A e_{q^{\prime}}^{(l)}, e_{q}^{(l)}\right\rangle\left\langle B e_{q}^{(l)}, e_{q^{\prime}}^{(l)}\right\rangle \psi^{k-l}(x, y) \frac{\varepsilon^{(k)}}{\varepsilon^{(l) 2}}\left|q^{l}\right|^{2}\left|q^{\prime l}\right|^{2} \frac{k^{n} \omega^{n}}{n !} \\
\quad=\int_{M} h_{y}^{(l)}\left(B e_{q}^{(l)}, A^{*} e_{q}^{(l)}\right) \psi^{k-l}(x, y) \frac{\varepsilon^{(k)}}{\varepsilon^{(l) 2}}\left|q^{l}\right|^{2} \frac{k^{n} \omega^{n}}{n !}
\end{gathered}
$$

and thus

$$
\begin{aligned}
\mid \int_{M} \hat{A}(x, & y) \hat{B}(y, x) \psi^{k-l}(x, y) \varepsilon^{(k)} \frac{k^{n} \omega^{n}}{n !} \mid \\
& \leq \int_{M}\left|\hat{A}(x, y) \hat{B}(y, x) \psi^{k}(x, y) e^{(k)}\right| \frac{k^{n} \omega^{n}}{n !} \\
& \leq \frac{\varepsilon^{(k)}}{\left(\varepsilon^{(l)}\right)^{2}}\left|q^{l}\right|^{2} k^{n} \int_{M}\left|h_{y}^{(l)}\left(A B e_{q}^{(l)}, e_{q}^{(l)}\right)\right| \frac{\omega^{n}}{n !}
\end{aligned}
$$

which is clearly bounded.

The second step consists in localizing the integral (2.3) in a neighbourhood $V$ of $x$; or, more precisely, to define a neighbourhood $U$ of the diagonal in $M \times M$ such that, for any $x$ in $M, V=\{y \in M \mid(x, y) \in U\}$. This is done by means of a version of the Morse lemma which we take from Combet [4].

Proposition 2. Let $(M, \omega, J)$ be a compact Kähler manifold and let $g$ denote its metric. Let $V$ be an open neighbourhood of the zero-section of the tangent bundle $p: T M \rightarrow M$, such that the map $\alpha: V \rightarrow M \times M, X \mapsto$ $\left(p(X), \exp _{p(X)} X\right)$ is well defined. Let $(L, \nabla, h)$ be a regular quantization bundle over $M$ and let $\psi$ be the corresponding 2-point function on $M \times M$. Then 
there exists an open neighbourhood $W$ of the zero-section in $T M$, and a smooth embedding $\nu: W \rightarrow T M$ such that

$$
(-\log \psi \circ \alpha \circ \nu)(X)=\frac{\pi}{2} g_{p(X)}(X, X), \quad X \in W .
$$

Proof. By compactness of $M$, there exists an open neighbourhood $V_{1}$ of the zero section of $T M$ and an open neighbourhood $U_{1}$ of the diagonal $\Delta$ in $M \times M$ such that

(i) $V_{1} \subset V$;

(ii) $\alpha_{\mid V_{1}}: V_{1} \rightarrow U_{1}$ is a smooth diffeomorphism;

(iii) $\bar{U}_{1} \cap \psi^{-1}(0)=\varnothing$.

Denote by $f: V_{1} \rightarrow \mathbb{R}$ the smooth function $f=-\log \psi \circ \alpha$. Observe that $f\left(0_{x}\right)=-\log \psi(x, x)=0$. If we denote by a subscript 2 differentiation in the vertical directions in $T M$ one has

$$
\left(D_{2} f\right)_{0_{x}}=\frac{1}{\psi(x, x)}\left(D_{2} \psi\right)_{(x, x)}=0
$$

since all points of the diagonal are critical points of $\psi$. Finally, using Proposition 4 of $\S 1$ we get $\left(\text { Hess }_{2} f\right)_{0_{x}}=\pi g_{x}$.

For $v \in V_{1}$, define the function $\tilde{g}_{v}:[0,1] \rightarrow \mathbb{R}: t \rightarrow f(t v)$. Clearly

$$
\tilde{g}_{v}(0)=0, \quad \tilde{g}_{v}^{\prime}(0)=\left(D_{2} f\right)_{0} v=0
$$

and

$$
\tilde{g}_{v}^{\prime \prime}(0)=\left(\operatorname{Hess}_{2} f\right)_{0}(v, v)=\pi g_{x}(v, v)>0
$$

whatever $v$ we choose. Taylor's formula with remainder gives us

$$
f(v)=\int_{0}^{1}(1-s) \tilde{g}_{v}^{\prime \prime}(s) d s
$$

and one sees that

$$
\tilde{g}_{v}^{\prime}(s)=\left(D_{2} f\right)_{s v} v, \quad \tilde{g}_{v}^{\prime \prime}(s)=\left(\operatorname{Hess}_{2} f\right)_{s v}(v, v) .
$$

We can thus introduce on each tangent space $M_{x}$ a family of symmetric bilinear forms, indexed by an element $v \in V_{1} \cap M_{x}$

$$
B_{v}\left(u, u^{\prime}\right)=\int_{0}^{1}(1-s)\left(\operatorname{Hess}_{2} f\right)_{s v}\left(u, u^{\prime}\right) d s .
$$

Clearly $B_{0}\left(u, u^{\prime}\right)=\frac{1}{2}\left(\operatorname{Hess}_{2} f\right)_{0}\left(u, u^{\prime}\right)=\frac{\pi}{2} g_{x}\left(u, u^{\prime}\right)$ is positive definite.

Using compactness again, we can find a neighbourhood $V_{2}$ of the zero-section in $T M$ such that (i) $V_{2} \subset V_{1}$; (ii) $B_{v}$ is positive definite for any $v \in V_{2}$. Recall that $f(v)=B_{v}(v, v)$. There exists a unique nonsingular element $C_{v}$ of $G L\left(M_{x}\right)$ which is symmetric relative to $B_{o}$ such that

$$
B_{v}\left(u, u^{\prime}\right)=B_{0}\left(C_{v} u, u^{\prime}\right)
$$

and all eigenvalues of $C_{v}$ are strictly positive. Furthermore the map $V_{2} \subset$ $T M \rightarrow \operatorname{End}(T M), v \mapsto C_{v}$ is smooth. Finally the endormorphism $C_{v}$ admits a unique symmetric, positive definite square root $C_{v}^{1 / 2}$ and

$$
B_{v}\left(u, u^{\prime}\right)=B_{0}\left(C_{v}^{1 / 2} u, C_{v}^{1 / 2} u^{\prime}\right) .
$$

Also $C_{0}^{1 / 2}=I$ and the map $v \rightarrow C_{v}^{1 / 2}$ is smooth. 
Define the map $\beta: V_{2} \rightarrow T M, v \mapsto C_{v}^{1 / 2} v$. This maps the zero-section onto the zero-section and one can find a neighbourhood $\widetilde{W}$ of the zero-section such that $\left.\beta\right|_{\widetilde{W}}: \widetilde{W} \rightarrow T M$ is an embedding. Clearly one may choose $W=\beta(\widetilde{W})$, $\nu=\left.\beta\right|_{W}{ }^{-1}$ and the proposition is proven.

Proposition 3. Let $(M, \omega, J)$ be a compact Kähler manifold, $(L, \nabla, h)$ be a quantization bundle for $M$ and $\psi$ be the corresponding 2-point function. Then for any $f$ belonging to $\hat{E}\left(L^{l}\right)$ the integral

$$
F_{k}(x)=\int_{M} f(x, y) \psi^{k}(x, y) k^{n} \frac{\omega^{n}}{n !}, \quad \text { for } k \geq l
$$

admits an asymptotic expansion

$$
F_{k}(x) \sim \sum_{r \geq 0} k^{-r} C_{r}(f)(x)
$$

where $C_{r}$ is a smooth differential operator depending only on the geometry of $M$.

Proof. Use Proposition 2 to construct a neighbourhood $U_{1}$ of the diagonal $\Delta$ in $M \times M$ and a neighbourhood $V_{1}$ of the zero section in $T M$ such that the following hold: (i) $\alpha: V_{1} \rightarrow U_{1}, X \mapsto\left(x, \exp _{x} X\right)$ is a smooth diffeomorphism;

(ii) $\exists \nu^{-1}: V_{1} \rightarrow \nu^{-1}\left(V_{1}\right) \subset T M$ a smooth embedding such that $-\log \psi \circ \alpha \circ \nu$ $=\frac{\pi}{2} g$ on $\nu^{-1}\left(V_{1}\right)$;

(iii) $\bar{U}_{1} \cap \psi^{-1}(0)=\varnothing$. Going back to the proof of Proposition 2, one observes that $\alpha \circ \nu: \nu^{-1}\left(V_{1}\right) \cap M_{x} \rightarrow M=\{x\} \times M$ is an embedding and hence one may define a nonzero smooth function $\theta$ by

$$
\left((\alpha \circ \nu)^{*} \frac{\omega^{n}}{n !}\right)(x, v)=\theta(x, v) d v
$$

where $d v$ denotes the linear Lebesgue measure on $M_{x}$. Shrinking $V_{1}$, if necessary, one may assume that $\theta$ is defined on $\bar{V}_{1}$ and hence is bounded as well as all its derivatives.

Choose an open neighbourhood $U_{2}$ of $\Delta$ in $M \times M$, with $\bar{U}_{2} \subset U_{1}$ and define $V_{2}=\alpha^{-1}\left(U_{2}\right)$. Let $\chi: M \times M \rightarrow[0,1]$ be a smooth function such that $\left.\chi\right|_{U_{2}}=1$ and supp $\chi \subset U_{1}$. Set $\eta=\max _{x, y \notin U_{2}} \psi(x, y)$. Clearly $\eta<1$ and $\psi(x, y) \leq \eta$ on $M \backslash U_{2}$. Let $U_{1, x}=\left\{y \in M \mid(x, y) \in U_{1}\right\}$, $U_{2, x}=\left\{y \in M \mid(x, y) \in U_{2}\right\}$ and $\chi_{x}(y)=\chi(x, y)$. The function $\chi_{x}$ is equal to 1 on $U_{2, x}$ and has support in $U_{1, x}$.

The function $f$ appearing in the statement of the proposition is a smooth function on $(M \times M) \backslash \psi^{-1}(0)$ (it may have singularities where $\psi$ vanishes). In particular it is well behaved in a neighbourhood of the diagonal. One has

$$
F_{k}(x)=\int_{U_{1, x}} f(x, y) \psi^{k}(x, y) k^{n} \frac{\omega^{n}}{n !}+\int_{M \backslash U_{1, x}} f(x, y) \psi^{k}(x, y) k^{n} \frac{\omega^{n}}{n !} .
$$

Going back to the Remark 1 of the previous section one sees that there exists a positive constant $C_{1}$ such that $\left|f \psi^{l}\right| \leq C_{1}$ on $M \times M$. Thus

$$
\left|\int_{M \backslash U_{1, x}} f(x, y) \psi^{k}(x, y) k^{n} \frac{\omega^{n}}{n !}\right| \leq C_{1} \eta^{k-l} k^{n} \operatorname{vol}_{M} .
$$


Also

$$
\begin{array}{rl}
\mid \int_{U_{1, x}} & f(x, y)\left(1-\chi_{x}(y)\right) \psi^{k}(x, y) k^{n} \frac{\omega^{n}}{n !} \mid \\
= & \left|\int_{U_{1, x} \backslash U_{2, x}} f(x, y)\left(1-\chi_{x}(y)\right) \psi^{k}(x, y) k^{n} \frac{\omega^{n}}{n !}\right| \\
\leq & C_{2} \eta^{k} k^{n} \operatorname{vol}_{M}
\end{array}
$$

if $|f| \leq C_{2}$ on $\bar{U}_{1}$. Grouping the terms we get

$$
\left|F_{k}(x)-\int_{U_{1, x}} \chi(x, y) f(x, y) \psi^{k}(x, y) k^{n} \frac{\omega^{n}}{n !}\right| \leq C \eta^{k} k^{n}, \quad \forall k \geq l
$$

where we have defined $C=\operatorname{vol}(M)\left(C_{1} \eta^{-l}+C_{2}\right)$. Thus this difference is exponentially uniformly small. The integral may be computed in the tangent space $M_{x}$ as

$$
\begin{aligned}
\int_{U_{1, x}} & \chi(x, y) f(x, y) \psi^{k}(x, y) k^{n} \frac{\omega^{n}}{n !} \\
= & \int_{V_{1, x}} \chi(\alpha \circ \nu)(x, v) f(\alpha \circ \nu)(x, v) e^{-\frac{k \pi}{2} g(v, v)} k^{n} \theta(x, v) d v
\end{aligned}
$$

where $V_{1, x}=(\alpha \circ \nu)^{-1} U_{1, x}$.

Denote by $G(x, v)$ the function on $T M$ defined by

$$
G(x, v)= \begin{cases}\chi((\alpha \circ \nu)(x, v)) f((\alpha \circ \nu)(x, v)) \theta(x, v), & \text { if }(x, v) \in V_{1} \\ 0, & \text { if }(x, v) \notin V_{1}\end{cases}
$$

It is smooth and compactly supported and

$$
\begin{gathered}
\int_{U_{1, x}} \chi(x, y) f(x, y) \psi(x, y) k^{n} \frac{\omega^{n}}{n !}=\int_{M_{x}} G(x, v) e^{-\frac{k \pi}{2} g(v, v)} k^{n} d v \\
=\int_{0}^{\infty} d r \int_{S_{x} M} G(x, r v) e^{-\frac{k \pi}{2} r^{2}} r^{2 n-1} k^{n} d v
\end{gathered}
$$

where $r(v)=g(v, v)^{1 / 2}$ and $S_{x} M$ is the unit sphere in $M_{x}$. Now use Taylor's formula with integral remainder for $G(x, r v)$

$$
G(x, r v)=\sum_{p=0}^{2 N} \frac{r^{p}}{p !}\left(D_{v}^{p} G\right)(x, 0)+r^{2 N+1} \int_{0}^{1} \frac{(1-s)^{2 N}}{(2 N) !}\left(D_{v}^{2 N+1} G\right)(x, r s v) d s .
$$

The integral of the remainder term is easily bounded since $G$ is compactly supported.

$$
\begin{aligned}
& \left|\int_{0}^{\infty} d r \int_{S_{x} M} d v k^{n} r^{2 n+2 N+2} \int_{0}^{1} d s \frac{(1-s)^{2 N}}{(2 N) !}\left(D_{v}^{2 N+1} G\right)(x, r s v) e^{-\frac{k \pi}{2} r^{2}}\right| \\
& =\mid k^{-N} \int_{0}^{\infty} d t \int_{S_{x} M} d v \int_{0}^{1} d s \frac{(2 t)^{n+N-\frac{1}{2}}}{\pi^{N+\frac{1}{2}}} \frac{(1-s)^{2 N}}{(2 N) !} \\
& \times\left(D_{v}^{2 N+1} G\right)\left(x, \sqrt{\frac{2 t}{k \pi}} s v\right) \frac{e^{-t}}{\sqrt{k}} \mid \\
& \leq k^{-N} \frac{C_{3}}{\sqrt{k}} .
\end{aligned}
$$


Observe finally that if $p$ is odd

$$
\int_{S_{x} M}\left(D_{v}^{p} G\right)(x, 0) d v=0
$$

since this is the integral of the restriction to the sphere of a homogeneous polynomial of odd degree. Putting these facts together we get

$$
\begin{aligned}
& k^{N}\left|F_{k}(x)-\sum_{p=0}^{N} \frac{(p+n-1) !}{(2 p) !}\left(\frac{2}{\pi}\right)^{p+n} \frac{k^{-p}}{2} \int_{S_{x} M}\left(D_{v}^{2 p} G\right)(x, 0) d v\right| \\
& \leq C \eta^{k} k^{n+N}+\frac{C_{3}}{\sqrt{k}} .
\end{aligned}
$$

This proves the proposition since the derivatives of the function $G$ in the vertical direction for $v=0$ do not depend on the choice of the cut-off function $\chi$, but depend only on $f$ and $\theta$ (which is related to the geometry alone).

Remark 4. If $A$ (resp. $B$ ) is an element of $\hat{E}\left(L^{l}\right)$ which corresponds to a rank one operator $A s=\langle s, u\rangle v ; u, v, s \in \mathscr{H}^{l}$ (resp. $B s=\langle s, \tilde{u}\rangle \tilde{v} ; \tilde{u}, \tilde{v}, s \in \mathscr{H}^{l}$ ) the formula $(2.3)$ for their $*$-product takes a very special form. Indeed

$$
\begin{aligned}
A(x, y) B(y, x) & =\frac{\overline{\left\langle u, e_{q^{\prime}}\right\rangle}\left\langle v, e_{q}\right\rangle \overline{\left\langle\tilde{u}, e_{q}\right\rangle}\left\langle\tilde{v}, e_{q^{\prime}}\right\rangle}{\left|\left\langle e_{q^{\prime}}, e_{q}\right\rangle\right|^{2}} \\
& =\frac{h_{x}(v(x), \tilde{u}(x)) h_{y}(\tilde{v}(y), u(y))}{\left(\varepsilon^{(l)}\right)^{2}} \frac{1}{\psi^{l}(x, y)} .
\end{aligned}
$$

Hence

$$
\left(A *_{k} B\right)(x)=\frac{h_{x}(v(x), \tilde{u}(x))}{\varepsilon^{(l)}} \int_{M} \frac{h_{y}(\tilde{v}(y), u(y))}{\varepsilon^{(l)}} \psi^{k-l}(x, y) e^{(k)} k^{n} \frac{\omega^{n}}{n !} .
$$

Thus it is the product of a symbol $\hat{C} \in \hat{E}\left(L^{l}\right),\left(C s=\langle s, \tilde{u}\rangle v ; \tilde{u}, v, s \in \mathscr{H}^{l}\right)$ by an integral of the form (2.5). Observe also that $k$ has been shifted by $l$.

Remark 5. Using Remark 4, we compute the first two terms of the asymptotic expansion of $A *_{k} B$ for $A$ and $B$ in $\hat{E}\left(L^{l}\right)$ and $k \geq l$.

$$
\begin{aligned}
\left(\hat{A} *_{k} \hat{B}\right)(x) \sim & (n-1) !\left(\frac{2}{\pi}\right)^{n} \frac{\varepsilon^{(k)}}{2} \int_{S_{x} M} G(x, 0) d v \\
& +\frac{n !}{2}\left(\frac{2}{\pi}\right)^{n+1} \frac{\varepsilon^{(k)}}{2} \frac{1}{k} \int_{S_{x} M} D_{v}^{2} G(x, 0) d v+\cdots
\end{aligned}
$$

where we still need to expand $\varepsilon^{(k)}$ and where

$$
\begin{aligned}
& G(x, 0)=\hat{A}(x) \hat{B}(x) \theta(x, 0), \\
& G(x, v)=\hat{A}(x, \exp h(v)) \hat{B}(\exp h(v), x) \theta(x, v) .
\end{aligned}
$$

Hence the first term is proportional to the product $\hat{A}(x) \hat{B}(x)$, the coefficient being

$$
\alpha=(n-1) !\left(\frac{2}{\pi}\right)^{n} \frac{1}{2} \theta(x, 0) \text { vol } S^{2 n-1} \varepsilon_{0}
$$

where $\varepsilon_{0}$ denotes the constant term in the asymptotic expansion of the rational function $\varepsilon^{(k)}$. Observe that one has the identity $1 *_{k} 1=1$ which tells us that 
$\alpha=1$. For the second term, we shall only compute its antisymmetric part. We have

$$
\begin{aligned}
& \left(\hat{A} *_{k} \hat{B}-\hat{B} *_{k} \hat{A}\right)(x) \\
& \quad \sim \frac{n !}{2}\left(\frac{2}{\pi}\right)^{n+1} \frac{\varepsilon_{0}}{2 k} \int_{S_{x} M}\left[D_{v}^{2} G_{(A, B)}(x, 0)-\left(D_{v}^{2} G\right)_{(B, A)}(x, 0)\right] d v+\cdots .
\end{aligned}
$$

Observe that one has the identities $1 *_{k} B=B *_{k} 1=B$ which imply that the above integrand reduces to

$$
\begin{aligned}
& \left(\hat{A} *_{k} \hat{B}-\hat{B} *_{k} \hat{A}\right)(x) \\
& \sim \frac{n !}{2}\left(\frac{2}{\pi}\right)^{n+1} \frac{\varepsilon_{0}}{k} \int_{S_{x} M}\left[D_{v, 2} \hat{A}(x, x) D_{v, 1} \hat{B}(x, x)\right. \\
& \text { - } \left.D_{v, 2} \hat{B}(x, x) D_{v, 1} \hat{A}(x, x)\right] \theta(x, 0) d v+\cdots
\end{aligned}
$$

where the indices 1,2 refer to the first (second) variable in a function of the form $\hat{A}(x, y)$. The integrand is a homogeneous polynomial of degree 2 which one integrates over the sphere $S_{x}^{2 n-1}$. Hence if one decomposes this polynomial into a multiple of $|v|^{2}$ and a harmonic polynomial, only the multiple of $|v|^{2}$ plays a role in the integration. Since $\hat{A}(x, y)$ is holomorphic in $x$ and antiholomorphic in $y$ one gets

$$
\left(\hat{A} *_{k} \hat{B}-\hat{B} *_{k} \hat{A}\right)(x) \sim \frac{\beta}{k}\{\hat{A}, \hat{B}\}(x)+\cdots
$$

where $\{$,$\} is the Poisson bracket of functions on M$ associated to $\omega$. From (2.10) and (2.11) one gets

$$
\beta=\frac{i}{2 \pi} \text {. }
$$

Remark 6. Going back to formula (2.9) applied to the product $*_{k}$ of elements $\hat{A}, \hat{B}$ of $\hat{E}\left(L^{l}\right)$ one sees that expanding the derivatives (compare 2.6) will give rise to bidifferential operators which are invariant under all the automorphisms of the quantization. Summarizing the above analysis we have

Theorem 1. Let $(M, \omega, J)$ be a compact Kähler manifold and $(L, \nabla, h)$ be a quantization bundle over $M$. Assume this quantization is regular (i.e. $\forall k \geq 1$, the function $\varepsilon^{(k)}$ corresponding to $L^{k}=\bigotimes^{k} L$ is a constant). Let $\hat{A}, \hat{B}$ be symbols of linear operators on $\mathscr{H}^{l}\left(=\right.$ space of holomorphic sections of $\left.L^{l}\right)$. Then the product $*_{k}$

$$
\left(\hat{A} *_{k} \hat{B}\right)(x)=\int_{M} \hat{A}(x, y) \hat{B}(y, x) \psi^{k}(x, y) \varepsilon^{(k)} k^{n} \frac{\omega^{n}}{n !}(y)
$$

defined for any $k \geq l$ admits an asymptotic expansion for $k$ tending to infinity

$$
\left(\hat{A} *_{k} \hat{B}\right)(x) \sim \sum_{r \geq 0} k^{-r} C_{r}(\hat{A}, \hat{B})(x)
$$

where the cochains $C_{r}$ are smooth bidifferential operators, invariant under the automorphisms of the quantization and determined by the geometry alone. Furthermore

$$
C_{0}(\hat{A}, \hat{B})(x)=\hat{A}(x) \hat{B}(x),
$$




$$
\frac{1}{2}\left(C_{1}(\hat{A}, \hat{B})-C_{1}(\hat{B}, \hat{A})\right)(x)=\frac{i}{4 \pi}\{\hat{A}, \hat{B}\}(x) .
$$

\section{A *-PRODUCT FOR FLAG MANIFOLDS}

We would like to show that the asymptotic expansion obtained above defines an associative formal *-product. For this we have, so far, only a proof when $(M, \omega, J)$ is a flag manifold.

Lemma 1. Let $(M, \omega, J)$ be a flag manifold with $M=G / K$ where $G$ is a compact simply-connected Lie group and $K$ the centralizer of a torus. Assume the geometric quantization conditions are satisfied and let $(L, \nabla, h)$ be a quantization bundle over $M$. Let $\mathscr{C}_{L}=\bigcup_{k} \hat{E}\left(L^{k}\right)$ be the union of the symbol spaces. Then $\mathscr{C}_{L}$ coincides with the space $E$ of vectors in $C^{\infty}(M)$ whose $G$-orbit is contained in a finite dimensional subspace.

Proof. Any symbol $\hat{A} \in \hat{E}\left(L^{l}\right)$ for some $l$. Its $G$-orbit is clearly contained in a finite dimensional subspace, namely $\hat{E}\left(L^{l}\right)$ itself. Hence $\mathscr{C}_{L} \subset E \subset C^{\infty}(M)$.

We have proven in [7] that $\mathscr{C}_{L}$ is dense in $C^{0}(M)$ for the topology of uniform convergence, hence is dense in $L^{2}(M)$ for the convergence in norm. Suppose $V$ is a finite dimensional invariant subspace of $L^{2}(M)$ then it is a direct sum of irreducible subspaces each of which is then closed in $L^{2}(M)$. Hence each irreducible subspace of $V$ must intersect $\mathscr{C}_{L}$. By invariance and irreducibility it is contained in $\mathscr{C}_{L}$. Thus $V$ and hence $E \subset \mathscr{C}_{L}$.

Corollary 1. If $\hat{A}, \hat{B}$ belong to $\hat{E}\left(L^{l}\right)$, there exists an integer a $(l)$ such that (i) $\hat{A} *_{k} \hat{B}$ belongs to $\hat{E}\left(L^{a(l)}\right)$; (ii) for every integer $r, C_{r}(\hat{A}, \hat{B})$ belongs to $\hat{E}\left(L^{a(l)}\right)$.

Proof. It was proved in Remark 2 of the previous section that the map $\hat{E}\left(L^{l}\right) \otimes$ $\hat{E}\left(L^{l}\right) \rightarrow C^{\infty}(M)$ given by $\hat{A} \otimes \hat{B} \mapsto \hat{A} *_{k} \hat{B}$ intertwines the action of $G$, hence (i). Similarly it was observed in Remark 6 that the bidifferential operators $C_{r}$ were invariant under $G$, hence (ii).

Consider now the asymptotic development given by Theorem 1:

$$
\hat{A} *_{k} \hat{B}=\sum_{r=0}^{N} k^{-r} C_{r}(\hat{A}, \hat{B})+R_{N}(\hat{A}, \hat{B}, k)
$$

where

$$
\lim _{k \rightarrow \infty} k^{N} R_{N}(\hat{A}, \hat{B}, k)=0
$$

uniformly in $x$. Corollary 1 tells us that $R_{N}(\hat{A}, \hat{B}, k)$ belongs to $\hat{E}\left(L^{a(l)}\right)$ where $a(l)$ is independent of $k$. Then

$$
\begin{aligned}
\left(\hat{A} *_{k} \hat{B}\right) *_{k} \hat{C}= & \sum_{r=0}^{N} k^{-r} C_{r}(\hat{A}, \hat{B}) *_{k} \hat{C}+R_{N}(\hat{A}, \hat{B}) *_{k} \hat{C} \\
= & \sum_{r, s=0}^{N} k^{-r-s} C_{s}\left(C_{r}(\hat{A}, \hat{B}), \hat{C}\right) \\
& +\sum_{r=0}^{N} k^{-r} R_{N}\left(C_{r}(\hat{A}, \hat{B}), \hat{C}, k\right)+R_{N}(\hat{A}, \hat{B}, k) *_{k} \hat{C} .
\end{aligned}
$$


The second term multiplied by $k^{N}$ clearly tends to zero when $k$ tends to infinity. For the third observe that we can write

$$
R_{N}(\hat{A}, \hat{B}, k)=\sum_{\alpha} u_{\alpha}(k) \hat{e}_{\alpha}
$$

where $\hat{e}_{\alpha}$ is a basis of $\hat{E}\left(L^{a(l)}\right)$ and where for each $\alpha, \lim _{k \rightarrow \infty} k^{N} u_{\alpha}(k)=0$. Now $\hat{e}_{\alpha} *_{k} \hat{C}$ is well defined and the limit for $k$ large is the usual pointwise product. Hence $\lim _{k \rightarrow \infty} k^{N} R_{N}(\hat{A}, \hat{B}, k) *_{k} \hat{C}=0$. This implies that the formal series $\sum_{r \geq 0} C_{r}(\hat{A}, \hat{B}) k^{-r}$ defines an associative deformation on $\mathscr{C}_{L}$. Hence we have proved the following theorem.

Theorem 2. The asymptotic expansion $\sum_{r \geq 0} k^{-r} C_{r}(u, v)$ defines a formal associative deformation of the usual product of functions in $\mathscr{C}_{L}$.

Remark. This is called a formal *-product since the antisymmetrization of the second term is the Poisson bracket of the functions $u$ and $v$. The product extends to all of $C^{\infty}(M)$, using uniform convergence.

\section{CONVERGENCE OF THE *-PRODUCT FOR HERMITIAN SYMMETRIC SPACES}

We prove that $\hat{A} *_{k} \hat{B}$ is a rational function of $k$ with no pole at infinity, when the flag manifold is a hermitian symmetric space. This implies that the asymptotic expansion converges for $k$ sufficiently large. If we know already that $\hat{A} *_{k} \hat{B}$ is a rational function of $k$, then the existence of the asymptotic expansion says it is smooth at infinity and so has no pole there. Hence it is enough to establish the rationality. As the proof is relatively long we shall split it into a series of lemmas.

Lemma 1. To prove that $\hat{A} *_{k} \hat{B}$ is a rational function of $k$, for any pair of symbols $\hat{A}, \hat{B} \in \hat{E}\left(L^{l}\right),(l<k)$ it is enough to prove that

$$
\int_{M} \psi(w, y)^{p} \psi(y, z)^{q} \frac{\omega^{n}(y)}{n !}
$$

depends rationally on $p$.

Proof. Formula (2.3) tells us that

$$
\left(\hat{A} *_{k} \hat{B}\right)(x)=\int_{M} \hat{A}(x, y) \hat{B}(y, x) \psi^{k}(x, y) \varepsilon^{(k)} k^{n} \frac{\omega^{n}(y)}{n !}
$$

for $\hat{A}, \hat{B} \in \hat{E}\left(L^{l}\right),(l<k)$. Since $\mathscr{H}^{l}$ is finite dimensional it is enough to prove rationality in the particular case where $\hat{A}$ and $\hat{B}$ are rank one operators

$$
A s=\langle s, u\rangle v, \quad B s=\langle s, \tilde{u}\rangle \tilde{v} .
$$

Then

$$
\left(\hat{A} *_{k} \hat{B}\right)(x)=\frac{\left\langle e_{q}, \tilde{u}\right\rangle\left\langle v, e_{q}\right\rangle}{\left\|e_{q}\right\|^{2}} \int_{M} \frac{\left\langle e_{q^{\prime}}, u\right\rangle\left\langle\tilde{v}, e_{q^{\prime}}\right\rangle}{\left\|e_{q^{\prime}}\right\|^{2}} \psi^{k-l}(x, y) \varepsilon^{(k)} k^{n} \frac{\omega^{n}(y)}{n !}
$$

where $\pi(q)=x, \pi\left(q^{\prime}\right)=y$. The term outside the integral is the symbol of an operator and belongs to $\hat{E}\left(L^{l}\right)$; it is independent of $k$.

There exists a basis of $\mathscr{H}^{l}$ composed of coherent states and so we may write

$$
u=\sum_{i \leq N} u_{i} e_{q_{i}}, \quad \tilde{v}=\sum_{i \leq N} v_{i} e_{q_{i}}
$$


and thus the integral reduces to

$$
\sum_{i, j \leq N} \bar{u}_{i} v_{j} \int_{M} \frac{\left\langle e_{q^{\prime}}, e_{q_{i}}\right\rangle\left\langle e_{q_{j}}, e_{q^{\prime}}\right\rangle}{\left\|e_{q^{\prime}}\right\|^{2}} \psi^{k-l}(x, y) \varepsilon^{(k)} k^{n} \frac{\omega^{n}(y)}{n !} .
$$

Assume we have proven that

$$
G(k, x, w)=\int_{M} \frac{\left\langle e_{q^{\prime}}, e_{q^{\prime \prime}}\right\rangle\left\langle e_{q^{\prime \prime}}, e_{q^{\prime}}\right\rangle}{\left\|e_{q^{\prime}}\right\|^{2}} \psi^{k-l}(x, y) \varepsilon^{(k)} k^{n} \frac{\omega^{n}(y)}{n !}
$$

(where $\pi\left(q^{\prime \prime}\right)=w$ ) depends rationally on $k$. Since $G$ depends in a real analytic way on $w$, and admits a unique analytic extension to $M \times M$, this analytic extension still depends in a rational way on $k$. Thus rationality of $G(k, x, w)$ is sufficient. Notice that

$$
G(k, x, w)=\int_{M} \psi^{l}(y, w) \psi^{k-l}(x, y) \varepsilon^{(k)} k^{n} \frac{\omega^{n}(y)}{n !}\left\|e_{q^{\prime \prime}}\right\|^{2} .
$$

Since $\left\|e_{q^{\prime \prime}}\right\|^{2}$ is independent of $k$ and $\varepsilon^{(k)}$ is a rational function of $k$, the rationality of $G$ is equivalent to the rationality of

$$
\int_{M} \psi^{l}(y, w) \psi^{k-l}(x, y) \frac{\omega^{n}(y)}{n !} .
$$

Set $p=k-l, q=l$ and

$$
H(p, q ; x, w)=\int_{M} \psi^{p}(x, y) \psi^{q}(y, w) \frac{\omega^{n}(y)}{n !}=H(q, p ; w, x) .
$$

It follows that if we prove the rationality of $H(p, q ; x, w)$ with respect to $p$ then we have proved the rationality with respect to $k$ of the product $*_{k}$.

Lemma 2. To prove that $H(p, q ; x, w)$ is rational in $p$ for a flag manifold $M=G / K \quad(G$ a compact Lie group, $K$ the centralizer of a torus in $G)$ it is enough to consider $G$ simple.

Proof. Flag manifolds are simply connected and one may also assume that the compact group $G$ is simply connected. Hence $G=G_{1} \times \cdots \times G_{r}, K=K_{1} \times$ $\cdots \times K_{r}$ and $M=G_{1} / K_{1} \times \cdots \times G_{r} / K_{r}=M_{1} \times \cdots \times M_{r}$. We shall denote by $p_{j}: M \rightarrow M_{j}$ the canonical projection. Let $\chi$ be the character of $K$ defining the line bundle $L=G \times_{\chi} \mathbb{C}$ and let $\chi_{j}$ be the restriction of $\chi$ to $K_{j}$. Denote by $L_{j}=G_{j} \times_{\chi_{j}} \mathbb{C}$ the corresponding line bundle on $M_{j}$ and consider $p_{j}^{*} L_{j}$, the pull-back bundle. $L$ is isomorphic to $\bigotimes_{j} p_{j}^{*} L_{j}$, hence

$$
\psi_{L}=\prod_{j} \psi_{p_{j}^{*}} L_{j}=\prod_{j}\left(\psi_{L_{j}} \circ p_{j}\right)
$$

Since the volume form $\frac{\omega^{n}}{n !}$ is a product of volume forms $\omega_{j}$ on each of the factors $M_{j}$ one sees that

$$
H(p, q ; x, w)=\prod_{j} \int_{M_{j}} \psi_{L_{j}}^{p}\left(p_{j}(x), p_{j}(y)\right) \psi_{L_{j}}^{q}\left(p_{j}(y), p_{j}(w)\right) \omega_{j}
$$

and the lemma is proven.

Let us recall some basic facts about hermitian symmetric spaces. Let $M=$ $G / K$ be a compact irreducible hermitian symmetric space. Then $M$ is simply 
connected [8, p. 376], and we may take $G$ to be connected, compact, simple with trivial centre and $K$ to be a maximal connected proper subgroup of $G$ [8, p. 382]. Furthermore the centre $Z(K)$ of $K$ is isomorphic to $U(1)$ [8, p. 382]. Let $\pi: G \rightarrow M$ be the canonical projection and let $0=\pi(e)$ denote the identity coset. The symmetry $s_{0}$ belongs to $Z(K)$ [8, p. 375]. Let $\mathfrak{g}$ denote the Lie algebra of $G$ and $\mathfrak{k}$ be the Lie algebra of $K$. Let $\sigma$ denote the involutive automorphism of $\mathfrak{g}$ defined by Ad $s_{0}$ and let $\mathfrak{g}=\mathfrak{k} \oplus \mathfrak{p}$ be the decomposition of $\mathfrak{g}$ into the +1 and -1 eigenspaces of $\sigma$. Let $\mathfrak{h}$ be a maximal abelian subalgebra of $\mathfrak{k}, \mathfrak{h}$ contains $\mathfrak{z}$, the Lie algebra of $Z(K)$ and is a maximal abelian subalgebra of $\mathfrak{g}$. Hence if $\mathfrak{g}^{\mathbb{C}}=\mathfrak{k}^{\mathbb{C}} \oplus \mathfrak{p}^{\mathbb{C}}$ is the complexified algebra of $\mathfrak{g}$, the subalgebra $\mathfrak{h}^{\mathbb{C}}$ is a Cartan subalgebra of $\mathfrak{g}^{\mathbb{C}}$. Let $\Delta$ be the set of roots of $\mathfrak{g}$ with respect to $\mathfrak{h}^{\mathbb{C}}$ and let $\Delta_{c}$ be the set of roots whose retrictions to ${ }^{\mathbb{C}}$ do not vanish identically. If $\Delta_{1}=\Delta \backslash \Delta_{c}$ then

$$
\mathfrak{k}^{\mathbb{C}}=\mathfrak{h}^{\mathbb{C}} \oplus \sum_{\beta \in \Delta_{l}} \mathfrak{g}^{\beta}, \quad \mathfrak{p}^{\mathbb{C}}=\sum_{\beta \in \Delta_{c}} \mathfrak{g}^{\beta} .
$$

The roots take real values on $i \mathfrak{h}$. Choose compatible Weyl chambers in $(i \mathfrak{h})^{*}$ and $(i \mathfrak{z})^{*}$ and denote by $\Delta^{+}$the corresponding positive roots. Let

$$
\mathfrak{n}_{+}=\sum_{\beta \in \Delta_{c}^{+}} \mathfrak{g}^{\beta}, \quad \mathfrak{n}_{-}=\sum_{-\beta \in \Delta_{c}^{+}} \mathfrak{g}^{\beta} .
$$

Then $\mathfrak{n}_{+}$and $\mathfrak{n}_{-}$are abelian subalgebras of $\mathfrak{p}^{\mathbb{C}}$ and $\mathfrak{p}^{\mathbb{C}}=\mathfrak{n}_{+} \oplus \mathfrak{n}_{-}[8$, p. 384].

Two roots $\alpha, \beta \in \Delta$ are said to be strongly orthogonal if neither $\alpha+\beta$ nor $\alpha-\beta$ belongs to $\Delta$. Let $\mathfrak{a}$ be a maximal abelian subalgebra of $\mathfrak{p}$ and let $r$ be its dimension. The number $r$ is called the rank of the symmetric space $M$. One proves $\left[8\right.$, p. 385] that there exists a subset $\left\{\gamma_{1}, \ldots, \gamma_{r}\right\}$ of $\Delta_{c}^{+}$ consisting of strongly orthogonal roots. If $\alpha \in \Delta$ let $h_{\alpha} \in \mathfrak{h}^{\mathbb{C}}$ be the element such that $B\left(h, h_{\alpha}\right)=\alpha(h)$ where $B$ is the Killing form of $\mathfrak{g}^{\mathbb{C}}$. There exist vectors $X_{\alpha} \in \mathfrak{g}^{\alpha}$ such that for all $\alpha \in \Delta,\left(X_{\alpha}-X_{-\alpha}\right)$ and $i\left(X_{\alpha}+X_{-\alpha}\right)$ belong to $\mathfrak{g}$. Furthermore

$$
\left[X_{\alpha}, X_{-\alpha}\right]=\frac{2}{\alpha\left(h_{\alpha}\right)} h_{\alpha} .
$$

Then we can choose the subalgebra $a$ so that it is spanned by the vectors $i\left(X_{\gamma_{j}}+X_{-\gamma_{j}}\right),(j \leq r)$.

Let $G^{\mathbb{C}}$ be the simply-connected Lie group with algebra $\mathfrak{g}^{\mathbb{C}}$ and let $N^{+}$, $K^{\mathbb{C}}, N^{-}$denote the analytic subgroups of $G^{\mathbb{C}}$ with algebras $\mathfrak{n}_{+}, \mathfrak{k}^{\mathbb{C}}, \mathfrak{n}_{-}$respectively. The following facts are standard:

(i) The exponential map induces a diffeomorphism of $\mathfrak{n}_{+}$(respectively $\mathfrak{n}_{-}$) onto $N^{+}$(respectively $N^{-}$), [8, p. 388].

(ii) The map $N_{-} \times K^{\mathbb{C}} \times N_{+} \rightarrow G^{\mathbb{C}},\left(n_{-}, k, n_{+}\right) \mapsto n_{-} k n_{+}$is a smooth diffeomorphism onto an open submanifold of $G^{\mathbb{C}}$ containing $G$, [8, p. 388].

(iii) The map $f: G / K \rightarrow G^{\mathbb{C}} / K^{\mathbb{C}} N_{+}, g K \mapsto g K^{\mathbb{C}} N_{+}$is a holomorphic diffeomorphism, [8, p. 393].

(iv) The map $\xi: \mathfrak{n}_{-} \rightarrow M, Y \mapsto \exp Y \cdot 0$ is a holomorphic diffeomorphism of $\mathfrak{n}_{-}$onto an open dense subset of $M,[8$, p. 395].

Let us now go back to the quantization bundle $L \rightarrow M$; as a homogeneous line bundle $L=G \times_{\chi} \mathbb{C}$ where $\chi$ is a character of $K$. Observe that $\chi$ extends holomorphically to $K^{\mathbb{C}}$ and trivially to $N_{+}$and thus defines a character of the parabolic $K^{\mathbb{C}} N_{+}$. One can view $L$ holomorphically as $L=G^{\mathbb{C}} \times_{\chi} \mathbb{C}$. 
Denote by $g \rightarrow \bar{g}$ the complex conjugation of $G^{\mathbb{C}}$ with respect to the real form $G$. If $q \in L^{0}$ ( $=L \backslash$ zero-section) and if $e_{q}$ is the corresponding coherent state one has

$$
g \cdot e_{q}=e_{\bar{g} q} \cdot
$$

Indeed the representation of $G$ on $\mathscr{H}$ extends to $G^{\mathbb{C}}$. Formula (4.1) is valid for $g$ in $G$, and both sides depend holomorphically on $g$. Hence (4.1) is valid for $G^{\mathbb{C}}$.

If $q_{0}$ is a nonzero element of $L_{0}$ (= fibre above 0$)$, the coherent state $e_{q_{0}}$ is a lowest weight vector for the representation of $G^{\mathbb{C}}$ on $\mathscr{H}$. Indeed if $n \in N_{-}$ and $h \in K^{\mathrm{C}}$ then

$$
\begin{gathered}
n \cdot e_{q_{0}}=e_{\bar{n} q_{0}}=e_{q_{0}}, \\
h \cdot e_{q_{0}}=e_{h q_{0}}=e_{\chi(h) q_{0}}=\overline{\chi(\bar{h})^{-1}} e_{q_{0}}=\chi(h) e_{q_{0}} .
\end{gathered}
$$

From its definition the 2-point function $\psi(x, y)$ is invariant under $G$ (i.e. $\psi(g x, g y)=\psi(x, y))$. This implies that in the integral given in Lemma 1 one can choose $x=0$. Also by the observation (iv) above one can assume that $y \in \xi\left(\mathfrak{n}_{-}\right)$. We thus compute for any $n \in N_{-}$

$$
\begin{gathered}
\psi(0, n \cdot 0)=\frac{\left|\left\langle e_{q_{0}}, e_{n q_{0}}\right\rangle\right|^{2}}{\left\|e_{q_{0}}\right\|^{2}\left\|e_{n q_{0}}\right\|^{2}}, \\
\left\langle e_{q_{0}}, e_{n q_{0}}\right\rangle=\left\langle e_{q_{n}}, \bar{n} e_{q_{0}}\right\rangle=\left\langle n^{-1} e_{q_{0}}, e_{q_{0}}\right\rangle=\left\|e_{q_{0}}\right\|^{2}
\end{gathered}
$$

using the same argument as for formula (4.1). Hence

$$
\psi(0, n \cdot 0)=\frac{\left\|e_{q_{0}}\right\|^{2}}{\left\|e_{n q_{0}}\right\|^{2}} \text {. }
$$

Similarly, if $z \in \xi\left(\mathfrak{n}_{-}\right)$and if $s_{0}$ is a holomorphic section of $L^{0}$ over $\xi\left(\mathfrak{n}_{-}\right)$

$$
\psi(n \cdot 0, z)=\frac{\left|\left\langle e_{n q_{0}}, e_{s_{0}(z)}\right\rangle\right|^{2}}{\left\|e_{n q_{0}}\right\|^{2}\left\|e_{s_{0}(z)}\right\|^{2}}=\frac{\left|\left\langle\bar{n} \cdot e_{q_{0}}, e_{s_{0}(z)}\right\rangle\right|^{2}}{\left\|e_{n q_{0}}\right\|^{2}\left\|e_{s_{0}(z)}\right\|^{2}} .
$$

Thus

$$
\int_{M} \psi(0, y)^{p} \psi(y, z)^{q} \frac{\omega^{n}(y)}{n !}=\int_{\xi\left(\mathrm{n}_{-}\right)} \frac{\left|\left\langle\bar{n} \cdot e_{q_{0}}, e_{s_{0}(z)}\right\rangle\right|^{2 q}\left\|e_{q_{0}}\right\|^{2 p}}{\left\|e_{n q_{0}}\right\|^{2(p+q)}\left\|e_{s_{0}(z)}\right\|^{2 q}} \frac{\omega^{n}(y)}{n !} .
$$

Observe that one can choose $q_{0}$ such that $\left\|e_{q_{0}}\right\|^{2}=1$. The above integral is the product of a function of $z$, independent of $p$ together with

$$
\int_{\xi\left(\mathfrak{n}_{-}\right)} \frac{\left|\left\langle\bar{n} \cdot e_{q_{0}}, e_{s_{0}(z)}\right\rangle\right|^{2 q}}{\left\|\bar{n} \cdot e_{q_{0}}\right\|^{2(p+q)}} \frac{\omega^{n}(n \cdot 0)}{n !} .
$$

Since the group $N_{+}$is nilpotent, the numerator of the integrand is a polynomial function on $\mathfrak{n}_{-}$.

We now wish to write this integral over $\xi\left(\mathfrak{n}_{-}\right)$as an integral over $\mathfrak{n}_{-}$, by making the change of variables explicit. Let $d z=d z_{1} \wedge \cdots \wedge d z_{n}$ be the $n$-form on $\mathfrak{n}_{-}\left(n=\operatorname{dim}_{\mathbb{C}_{-}}\right)$which is translation invariant. This induces an invariant form, still denoted by $d z$, on $N_{-}$. Clearly $\xi^{-1 *}(d z \wedge d \bar{z})$ is a volume form on $\xi\left(\mathfrak{n}_{-}\right)$and so has the form

$$
\xi^{-1 *}(d z \wedge d \bar{z})=f \frac{\omega^{n}(z)}{n !}
$$


for some function $f$. Since $G$ acts transitively on $M$, any element $g \in G^{\mathbb{C}}$ can be written in the form $g=u(g) k(g) n_{+}(g)$ where $u(g) \in G, k(g) \in K^{\mathbb{C}}$, $n_{+}(g) \in N_{+}$. In particular for $n \in N_{-}$

$$
\left.\xi^{-1 *}(d z \wedge d \bar{z})\right|_{n \cdot 0}=f(n \cdot 0) \frac{\omega^{n}(u(n) \cdot 0)}{n !}=f(n \cdot 0) \frac{\omega^{n}(0)}{n !} \circ u(n)_{*}^{-1}
$$

or equivalently

$$
\left(\xi^{-1 *} d z\right)_{n \cdot 0} \circ u(n)_{*} \wedge\left(\xi^{-1 *} d \bar{z}\right)_{n \cdot 0} \circ u(n)_{*}=\frac{f(n \cdot 0)}{f(0)}\left(\xi^{-1 *} d z\right)_{0} \wedge\left(\xi^{-1 *} d \bar{z}\right)_{0}
$$

But

$$
\begin{aligned}
\left(\xi^{-1 *} d z\right)_{n \cdot 0} \circ u(n)_{*} & =\left(\xi^{-1 *} d z\right)_{n \cdot 0} \circ\left(n n_{+}(n)^{-1} k(n)^{-1}\right)_{*} \\
& =\left(\xi^{-1 *} d z\right)_{0} \circ\left(n_{+}(n)^{-1} k(n)^{-1}\right)_{*}
\end{aligned}
$$

and since the action of $N_{+}$is unipotent, one has

$$
\frac{f(n \cdot 0)}{f(0)}=\left|\operatorname{det}\left(\mathrm{Ad}_{n_{-}} k(n)^{-1}\right)\right|^{2} \text {. }
$$

One can choose normalizations such that $f(0)=1$. After this change of variable the integral becomes

$$
\int_{\mathfrak{n}_{-}} \frac{\left|\left\langle\bar{n} e_{q_{0}}, e_{s_{0}(z)}\right\rangle\right|^{2 q}}{\left\|\bar{n} e_{q_{0}}\right\|^{2(p+q)}}\left|\operatorname{det}\left(\operatorname{Ad}_{n_{-}} k(n)^{-1}\right)\right|^{-2} d z \wedge d \bar{z} .
$$

We still wish to write the denominator and the jacobian more explicitly. Observe that

implies

$$
n=u(n) k(n) n_{+}(n)
$$

$$
\bar{n}=u(n) \overline{k(n)} \overline{n_{+}(n)}
$$

Since conjugation permutes $N_{+}$and $N_{-}$and since $e_{q_{0}}$ is a lowest weight vector

$$
\left\|\bar{n} \cdot e_{q_{0}}\right\|^{2}=\left\|\overline{k(n)} e_{q_{0}}\right\|^{2}=|\chi(\overline{k(n)})|^{2}\left\|e_{q_{0}}\right\|^{2}=\left|\chi\left(k(n)^{-1}\right)\right|^{2} .
$$

Since the centre of $K^{\mathbb{C}}$ is one dimensional, all characters of $K^{\mathbb{C}}$ are powers of a given one $\mu$, which we choose in such a way that $\chi\left(k^{-1}\right)=\mu(k)^{a}$ for some positive integer a.

Clearly $k \mapsto \operatorname{det}\left(\operatorname{Ad}_{n_{-}} k^{-1}\right)$ is also a character of $K^{\mathbb{C}}$ and thus there exists an integer $a^{\prime}$ such that

$$
\operatorname{det}\left(\operatorname{Ad}_{n_{-}} k(n)^{-1}\right)=\mu(k(n))^{a^{\prime}} .
$$

We conclude this discussion with the following lemma.

Lemma 3. To prove that $H(p, q ; x, w)$ is rational in $p$ for an irreducible hermitian symmetric space $M=G / K$ it is enough to prove that

$$
\int_{\mathfrak{n}_{-}} \frac{\left|\left\langle\bar{n} e_{q_{0}}, e_{s_{0}(z)}\right\rangle\right|^{2}}{|\mu(k(n))|^{2 p}} d z d \bar{z}
$$

is rational in $p$. Here the numerator is a polynomial on $n_{-}$and $\mu$ is the fundamental character of $K$.

Proof. Combining the preceding arguments, we see that the rationality of $H(p, q ; x, w)$ is equivalent to the rationality of

$$
\int_{n_{-}} \frac{\left|\left\langle\bar{n} e_{q_{0}}, e_{s_{0}(z)}\right\rangle\right|^{2}}{|\mu(k(n))|^{2\left(a p+a q+a^{\prime}\right)}} d z d \bar{z}
$$

with respect to $p$. Replacing $a p+a q+a^{\prime}$ by $p$ will not change the question. 
Since $\mathfrak{n}_{-}$is isomorphic (as a vector space) to $\mathfrak{p}$, this integral may be viewed as an integral on $\mathfrak{p}$. Our next aim is to decompose this integral into "radial" and "angular" integrals, but first we introduce some more notation. Let $m$ be the centralizer of $\mathfrak{a}$ in $\mathfrak{k}$, and let $M$ be the corresponding connected subgroup of $K$. Let $\mathfrak{t}$ be a maximal abelian subalgebra of $\mathfrak{g}$ containing $\mathfrak{a}$ and let $\mathfrak{t}^{\mathbb{C}}$ be the corresponding Cartan subalgebra of $\mathfrak{g}^{\mathbb{C}}$. Denote by $\widetilde{\Delta}$ the set of roots of $\mathfrak{g}^{\mathbb{C}}$ relative to $\mathfrak{t}^{\mathbb{C}}$, and by $\widetilde{\Delta}_{1}$ the set of roots in $\widetilde{\Delta}$ which do not vanish identically on $\mathfrak{a}$. As above the roots take real values on $i t^{*}$. Choose compatible Weyl chambers in $i t^{*}$ and in $i \mathfrak{a}^{*}$.

Consider the map $\beta: K / M \times \mathfrak{a} \rightarrow \mathfrak{p},(k M, a) \mapsto \operatorname{Ad} k(a)$. It is surjective. Now $K / M, \mathfrak{p}, \mathfrak{a}$ have invariant metrics induced by the Killing form of $\mathfrak{g}$. Denote by $d k_{M}$ and $d a$ the corresponding Riemannian measures on $K / M$ and $\mathfrak{a}$, respectively. The measure on $\mathfrak{p} \cong \mathfrak{n}_{-}$was already denoted $d z \wedge d \bar{z}$. Then we have the identity $[9$, p. 382]

$$
\beta^{*}(d z \wedge d \bar{z})_{(k M, a)}=\left|\prod_{\lambda \in \widetilde{\Delta}_{1}^{+}} \lambda(a)\right| d k_{M} d a
$$

and thus the following lemma holds.

Lemma 4. Let $F$ be a function on $\mathfrak{p}$ which is integrable. Then

$$
\int_{\mathfrak{p}} F(z) d z \wedge d \bar{z}=\int_{K / M} d k_{M} \int_{\mathfrak{a}}\left|\prod_{\lambda \in \widetilde{\Delta}_{1}^{+}} \lambda(a)\right| F(\operatorname{Ad} k(a)) d a
$$

and in (4.2) one can replace the integral over $K / M$ by an integral over $K$, introducing some normalization constant $C$.

Consider, as above, the vectors $X_{j}=i\left(X_{\gamma_{j}}+X_{-\gamma_{j}}\right),(j \leq r)$, which form a basis of $\mathfrak{a}$ and also the vectors $Y_{j}=X_{\gamma_{j}}-X_{-\gamma_{j}}$ and $H_{j}=2 i h_{j} / B\left(\gamma_{j}, \gamma_{j}\right)$. They determine $r$ mutually commuting $s u(2)$-subalgebras of $\mathfrak{g}$

$$
\left[X_{j}, Y_{j}\right]=-2 H_{j}, \quad\left[H_{j}, X_{j}\right]=-2 Y_{j}, \quad\left[H_{j}, Y_{j}\right]=2 X_{j} .
$$

This implies that

$$
\operatorname{Ad} \exp \frac{\pi}{4} Y_{j}\left(X_{j}\right)=H_{j}, \quad \operatorname{Ad} \exp \frac{\pi}{4} Y_{j}\left(H_{j}\right)=-X_{j}
$$

In particular the automorphism $\tau$ of $\mathfrak{g}$ defined by

$$
\tau=\operatorname{Ad} \exp \frac{\pi}{4} Y_{1} \cdots \operatorname{Ad} \exp \frac{\pi}{4} Y_{r}
$$

sends the algebra $\mathfrak{a}$ onto the subalgebra $\mathfrak{l}$ of $\mathfrak{h}$ spanned by the $H_{j}$ 's, $(j \leq r)$ and sends $\mathfrak{l}$ onto $\mathfrak{a}$. The system of roots of $\mathfrak{g}^{\mathbb{C}}$ relative to $\mathfrak{t}^{\mathbb{C}}$ corresponds to the system of roots of $\mathfrak{g}^{\mathbb{C}}$ relative to $\mathfrak{h}^{\mathbb{C}}$. In particular the roots in $\widetilde{\Delta}_{1}$ correspond to the set $\Phi$ of roots in $\Delta$ which do not vanish identically on $\mathfrak{l}$. The roots in $\Phi$ come in pairs $\alpha,-\alpha$ and we shall denote by $\Phi^{+}$any subset of $\Phi$ obtained by choosing arbitrarily one root in each pair. Then

$$
\left|\prod_{\lambda \in \widetilde{\Delta}_{1}^{+}} \lambda(a)\right|=\left|\prod_{\rho \in \Phi^{+}} \rho(\tau(a))\right| .
$$


Lemma 5. (i) The function $k^{\prime} \mapsto \mu\left(k\left(\exp \operatorname{Ad} k^{\prime}(\tilde{A})\right)\right)$ is constant on $K$, where $\widetilde{A}$ denotes the $\mathfrak{n}_{-}$component of an element $A$ of $\mathfrak{a}$.

(ii) Any $K$-invariant polynomial on $\mathfrak{p}$ is necessarily of even degree when restricted to $\mathfrak{a}$.

Proof. (i)

$$
\exp \operatorname{Ad} k^{\prime}(\widetilde{A})=k^{\prime} \exp \widetilde{A} k^{\prime-1}=k^{\prime} u(\exp \tilde{A}) k(\exp \widetilde{A}) n_{+}(\exp \widetilde{A}) k^{\prime-1}
$$

and thus

$$
k\left(\exp \operatorname{Ad} k^{\prime}(\tilde{A})\right)=k^{\prime} k(\exp \widetilde{A}) k^{\prime-1}
$$

and (i) follows, since $\mu$ is a character of $K$.

(ii) If $P$ is the invariant polynomial and $A$ an element of $\mathfrak{a}$, write $A=$ $\sum_{j} a_{j} X_{j}$; then $P(A)$ is a polynomial in the $a_{j}$ 's. Observe that

$$
\begin{aligned}
& \text { Ad } \exp \pi H_{j}\left(X_{k}\right)=X_{k} \quad \text { if } k \neq j, \\
& \text { Ad } \exp \pi H_{j}\left(X_{j}\right)=-X_{j}
\end{aligned}
$$

and thus $P\left(a_{1}, \ldots, a_{r}\right)$ is even in each variable $a_{j}$.

Lemma 6. The function $\mu\left(k\left(\exp \sum_{j \leq r} a_{j} X_{-\gamma_{j}}\right)\right)$ is a product of $r$ functions each depending on only one of the $a_{j}$.

Proof. The subgroup exp $t X_{-\gamma_{j}}$ is contained in the $S L(2, \mathbb{C})$-subgroup of $G^{\mathbb{C}}$ whose Lie algebra is spanned by $X_{j}, Y_{j}, H_{j}$. Since these $r$ subgroups are commuting

$$
\exp \sum_{j \leq r} a_{j} X_{-\gamma_{j}}=\prod_{j \leq r} \exp a_{j} X_{-\gamma_{j}} .
$$

Write each of the terms as a product $\exp a_{j} X_{-\gamma_{j}}=u_{j} k_{j} n_{j}^{+}$with $u_{j} \in G$, $k_{j} \in K^{\mathbb{C}}, n_{j}^{+} \in N_{+}$and observe that each of these elements belongs to the corresponding commuting $S L(2, \mathbb{C})$-subgroups. Hence

$$
\prod_{j \leq r} u_{j} k_{j} n_{j}^{+}=\prod_{j \leq r} u_{j} \prod_{j \leq r} k_{j} \prod_{j \leq r} n_{j}^{+}
$$

and thus

$$
\mu\left(k\left(\exp \sum_{j \leq r} a_{j} X_{-\gamma_{j}}\right)\right)=\prod_{j \leq r} \mu\left(k_{j}\right)
$$

where each of the $k_{j}$ 's depends on $a_{j}$ alone. This proves the lemma.

If we set $Q(z)=\left|\left\langle\bar{n} e_{q_{0}}, e s_{0}(z)\right\rangle\right|^{2}$ then $Q(z)$ is a polynomial on $\mathfrak{n}_{-}$and we may summarize the above analysis by rewriting the integral appearing in Lemma 3 as

$$
\begin{aligned}
\int_{\mathfrak{n}_{-}} \frac{Q(z) d z \wedge d \bar{z}}{|\mu(k(n))|^{2 p}} & =\int_{K / M} d k_{M}^{\prime} \int_{\mathfrak{a}} d a \frac{\left|\prod_{\lambda \in \widetilde{\Delta}_{1}^{+}} \lambda(a)\right| Q\left(\operatorname{Ad} k^{\prime}(a)\right)}{\left|\mu\left(k\left(\exp \operatorname{Ad} k^{\prime}(a)\right)\right)\right|^{2 p}} \\
& =C \int_{\mathfrak{a}} \frac{\left|\prod_{\rho \in \Phi^{+}} \rho(\tau(a))\right|}{\left|\prod_{j \leq r} \mu\left(k_{j}\right)\right|^{2 p}} d a \int_{K} Q\left(\operatorname{Ad~} k^{\prime}(a)\right) d k^{\prime}
\end{aligned}
$$


where $d k^{\prime}$ is the Haar measure on $K$. The integral of $Q$ over $K$ gives a polynomial $\bar{Q}\left(a_{1}, \ldots, a_{r}\right)$ invariant under $K$. Thus it is of even degree in each $a_{j}$.

Lemma 7. The polynomial

$$
R\left(a_{1}, \ldots, a_{r}\right)=\left|\prod_{\rho \in \Phi^{+}} \rho\left(\tau\left(\sum_{j \leq r} a_{j} H_{j}\right)\right)\right|
$$

is of odd degree in each argument $a_{j}$.

Proof. To see this we fix some $j$ and partition $\Phi^{+}$

$$
\boldsymbol{\Phi}^{+}=\left\{\gamma_{j}\right\} \cup\left\{\rho \in \boldsymbol{\Phi}^{+} \mid \rho \perp \gamma_{j}\right\} \cup \Phi_{j}^{+}
$$

where the last set consists of all elements not in the first two sets. If $\rho$ is orthogonal to $\gamma_{j}$ then $\rho\left(\sum_{j} a_{j} H_{j}\right)$ is independent of $a_{j}$. Also $\gamma_{j}\left(\sum_{j} a_{j} H_{j}\right)=$ $a_{j} \gamma_{j}\left(H_{j}\right)$, so is of degree one. Finally, if $\rho \in \Phi_{j}^{+}$and $s_{j}$ denotes the element of the Weyl group associated to $\gamma_{j}$, then $s_{j} \rho$ differs from both $\pm \rho$ and one of $\pm s_{j} \rho$ is in $\Phi^{+}$. Since $s_{j}$ fixes all the $\gamma_{k}$ for $k \neq j$,

$$
\pm\left(s_{j} \rho\right)\left(\sum_{k} a_{k} H_{k}\right)= \pm \rho\left(-a_{j} H_{j}+\sum_{k \neq j} a_{k} H_{k}\right)
$$

and thus the root which belongs to $\Phi^{+}$actually is in $\Phi_{j}^{+}$. Hence $\Phi_{j}^{+}$has an even number of terms which pair off to contribute factors of the form

$$
\begin{gathered}
\left(\rho\left(\sum_{k \neq j} a_{k} H_{k}\right)-\rho\left(a_{j} H_{j}\right)\right)\left(\rho\left(\sum_{k \neq j} a_{k} H_{k}\right)+\rho\left(a_{j} H_{j}\right)\right) \\
=\rho\left(\sum_{k \neq j} a_{k} H_{k}\right)^{2}-\rho\left(a_{j} H_{j}\right)^{2} .
\end{gathered}
$$

The lemma now follows.

Lemma 8. The function $\left|\mu\left(k_{j}\right)\right|^{2}$ of $a_{j}$ is of the form $\left(1+a_{j}^{2}\right)^{\alpha}$ for some positive power $\alpha$.

Proof. The calculation can be done in a fixed $S L(2, \mathbb{C})$. We have

$$
X_{+}=\left(\begin{array}{ll}
0 & 1 \\
0 & 0
\end{array}\right) \in \mathfrak{n}_{+}, \quad X_{-}=\left(\begin{array}{ll}
0 & 0 \\
1 & 0
\end{array}\right) \in \mathfrak{n}_{-}
$$

and we decompose

$$
\exp a X_{-}=\left(\begin{array}{ll}
1 & 0 \\
a & 1
\end{array}\right)=u k n_{+}
$$

with $u \in S U(2), k$ diagonal and $n_{+}$upper triangular with $1 \mathrm{~s}$ on the diagonal. If

$$
k=\left(\begin{array}{cc}
c & 0 \\
0 & c^{-1}
\end{array}\right), \quad n_{+}=\left(\begin{array}{ll}
1 & z \\
0 & 1
\end{array}\right)
$$

with $c \in \mathbb{C}^{*}, z \in \mathbb{C}$ then multiplying out and solving gives

$$
|c|^{2}=1+a^{2} \text {. }
$$


The lemma follows since every character of the diagonal subgroup has the form

$$
\left(\begin{array}{cc}
c & 0 \\
0 & c^{-1}
\end{array}\right) \mapsto c^{\alpha} \text {. }
$$

If we expand the polynomial $R \bar{Q}$ in monomials, only odd powers will occur as a consequence of Lemma 7 . Using Lemma 8 , it follows that

$$
\int_{\mathfrak{a}}\left|\prod_{\rho \in \Phi^{+}} \rho(\tau(a))\right| \frac{\bar{Q}\left(a_{1}, \ldots, a_{r}\right)}{\left|\prod_{j \leq r} \mu\left(k_{j}\right)\right|^{2 p}} d a
$$

is a sum of terms each of which is a product of integrals of the form

$$
\int_{-\infty}^{\infty} \frac{\left|x^{2 s+1}\right|}{\left(1+x^{2}\right)^{\alpha p}} d x
$$

where $s$ is in a fixed range independent of $p$. A simple calculation yields the next lemma.

Lemma 9.

$$
\int_{-\infty}^{\infty} \frac{\left|x^{2 s+1}\right|}{\left(1+x^{2}\right)^{\alpha p}} d x=\frac{s !}{(\alpha p-1) \cdots(\alpha p-s-1)} .
$$

In particular the integral is a rational function of $p$ without pole at infinity.

This can be summarized in the following theorem.

Theorem. Let $M$ be a compact hermitian symmetric space and let $(L, \nabla, h)$ be a quantization bundle over $M$. Let $L^{k}=\bigotimes^{k} L$ and let $\mathscr{H}^{k}$ be the space of holomorphic sections of $L^{k}$. Let $\hat{E}\left(L^{k}\right)$ be the space of symbols of operators on $\mathscr{H}^{k}$. If $\hat{A}, \hat{B}$ belong to $\hat{E}\left(L^{l}\right)$ and $k \geq l$, the product $\hat{A} *_{k} \hat{B}$ depends rationally on $k$ and has no pole at infinity.

Corollary. The asymptotic expansion of $\hat{A} *_{k} \hat{B}$ is convergent.

Remark. A calculation along similar lines for the nonhermitian symmetric flag manifold $U(3) / U(1) \times U(1) \times U(1)$ leads to a similar result. It thus seems reasonable to suggest, in conclusion, the following conjecture.

Conjecture. For any generalized flag manifold the $*_{k}$-product of two symbols is a rational function of $k$ without pole at infinity.

\section{REFERENCES}

1. F. Bayen et al., Deformation theory and quantisation, Ann. Physics 111 (1978), 1-151.

2. F. A. Berezin, Quantisation of Kähler manifold, Comm. Math. Phys. 40 (1975), 153.

3. E. Calabi, Isometric imbeddings of complex manifolds, Ann. of Math. 58 (1953), 1-23.

4. E. Combet, Intégrales exponentielles, Lecture Notes in Math., vol. 937, Springer-Verlag, Berlin, Heidelberg and New York, 1982.

5. P. Griffiths and J. Harris, Principles of algebraic geometry, Wiley, New York, 1978.

6. C. Moreno, * products on some Kähler manifolds, Lett. Math. Phys. 11 (1986), 361-372.

7. M. Cahen, S. Gutt and J. Rawnsley, Quantisation of Kähler manifolds I: Geometric interpretation of Berezin's quantisation, J. Geom. Phys. 7 (1990), 45-62. 
8. S. Helgason, Differential geometry, Lie groups and symmetric spaces, Academic Press, New York, 1978.

9. __ Differential geometry and symmetric spaces, Academic Press, New York, 1962.

(Michel Cahen and Simone Gutt) Département de Mathématique, Ulb Campus Plaine CP 218, 1050 BrusSELS, BELGIUM

E-mail address, Simone Gutt: sgutt@tc1.vub.ac.be

(John Rawnsley) Mathematics Institute, University of Warwick, Coventry CV4 7AL, UK

E-mail address: jhr@maths.warwick.ac.uk 